\title{
ZERO-AUTOMATIC QUEUES AND PRODUCT FORM
}

\author{
THU-HA DAO-THI *** AND \\ JEAN MAIRESSE, ${ }^{* * * *}$ LIAFA
}

\begin{abstract}
We introduce and study a new model: zero-automatic queues. Roughly, zero-automatic queues are characterized by a special buffering mechanism evolving like a random walk on some infinite group or monoid. The salient result is that all stable zero-automatic queues have a product form stationary distribution and a Poisson output process. When considering the two simplest and extremal cases of zero-automatic queues, we recover the simple M/M/1 queue and Gelenbe's $G$-queue with positive and negative customers.
\end{abstract}

Keywords: Queueing theory; M/M/1 queue; G-queue; quasi-reversibility; product form; quasi-birth-and-death process

2000 Mathematics Subject Classification: Primary 60K25; 68M20

\section{Introduction}

In this section we provide an informal description of a special type of zero-automatic queue (corresponding to a free product of three finite monoids). Consider a queue with a single server and an infinite capacity buffer. Customers are colored either in red (R), blue (B), or green (G), with a finite set of possible shades available within each color: $\Sigma_{\mathrm{R}}, \Sigma_{\mathrm{B}}, \Sigma_{\mathrm{G}}$. In the buffer, two consecutive customers of the same color can either cancel each other or merge to create a new customer of the same color. Customers of different colors do not interact. This process is illustrated in Figure 1. The shades are modified in the merging procedure, according to the following internal law: $\Sigma_{i} \times \Sigma_{i} \rightarrow \Sigma_{i} \cup\{1\}, i \in\{\mathrm{R}, \mathrm{G}, \mathrm{B}\}$, with 1 coding for the cancellation. The only, but crucial, restriction is that each internal law should be associative.

We now give a more detailed account of the model and results. Zero-automatic queues may be viewed as the synthesis of a simple queue and a random walk on a zero-automatic pair. We first recall these last two models.

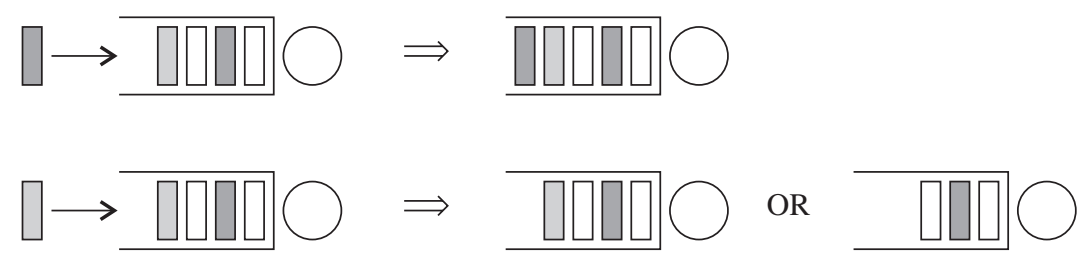

FIGURE 1: A zero-automatic queue.

Received 28 July 2005; revision received 19 April 2007.

* Postal address: LIAFA, CNRS-Université Paris 7, case 7014, 2 place Jussieu, 75251 Paris Cedex 05, France.

** Email address: daothi@liafa.jussieu.fr

*** Email address: mairesse@liafa.jussieu.fr 
The $\mathrm{M} / \mathrm{M} / 1 / \infty$ first-in-first-out (FIFO) queue, or simply referred to as an $\mathrm{M} / \mathrm{M} / 1$ queue, is the Markovian queue with arrivals and services occurring at constant rates, say $\lambda$ and $\mu$, a single server, an infinite capacity buffer, and a FIFO discipline. This is arguably the simplest and also the most studied model in queueing theory, with at least one book devoted to it [7]. The queue-length process is a continuous-time jump Markov process and its infinitesimal generator $Q$ is given by $Q(n, n+1)=\lambda, Q(n+1, n)=\mu$, for all $n \in \mathbb{N}$. Under the stability condition $\lambda<\mu$, the queue-length process is ergodic, and its stationary distribution $\pi$ is given by

$$
\pi(n)=\left(1-\frac{\lambda}{\mu}\right)\left(\frac{\lambda}{\mu}\right)^{n} .
$$

Besides, and this constitutes the celebrated Burke's theorem, the departure process in equilibrium has the same law as the arrival process.

Let us introduce the a priori completely unrelated model of a random walk on a plain group studied in [22] and [23]. Let $X$ be an infinite group or monoid with a finite set of generators $\Sigma$. Let $v$ be a probability measure on $\Sigma$ and let $\left(x_{i}\right)_{i \in \mathbb{N}}$ be a sequence of $\Sigma$-valued independent, identically distributed random variables (i.i.d. RVs) of law $v$. Let $\left(X_{n}\right)_{n}$ be the sequence of $X$-valued RVs defined by $X_{0}=1_{X}, X_{n+1}=X_{n} * x_{n}=x_{0} * x_{1} * \cdots * x_{n}$, where $1_{X}$ is the unit element of $X$ and $*$ is the group or monoid law. By definition, $\left(X_{n}\right)_{n}$ is a realization of the random walk $(X, v)$. Now we assume that the pair $(X, \Sigma)$ is formed by a plain monoid with natural generators, the definition of which is given in Section 2.2. For the moment, however, it suffices to say that the elements of $X$ can be set in bijection with a regular language $L(X, \Sigma) \subset \Sigma^{*}$. The random walk $\left(X_{n}\right)_{n}$ is viewed as evolving on $L(X, \Sigma)$. If $X_{n}=u a$, $u \in X, a \in \Sigma$, and $x_{n}=b \in \Sigma$, then

$$
X_{n+1}= \begin{cases}u & \text { if } a * b=1_{X}, \\ u c & \text { if } a * b=c \in \Sigma, \\ u a b & \text { otherwise. }\end{cases}
$$

Now we assume that the random walk is transient. Let $v^{\infty}\left(u \Sigma^{\mathbb{N}}\right)$ be the probability that the random walk goes to infinity in the 'direction' $u$ (i.e. $v^{\infty}\left(u \Sigma^{\mathbb{N}}\right)=\mathrm{P}$ there exists an $N$ for all $\left.\left.n \geq N, X_{n} \in u \Sigma^{*}\right\}\right)$. The following is the main result in [22]:

$$
v^{\infty}\left(u \Sigma^{\mathbb{N}}\right)=\hat{q}\left(u_{1}\right) \cdots \hat{q}\left(u_{n-1}\right) \hat{r}\left(u_{n}\right) \quad \text { for all } u=u_{1} \cdots u_{n} \in L(X, \Sigma),
$$

where $\hat{q}(a) \in(0,1)$ and $\hat{r}(a) \in(0,1)$, for all $a \in \Sigma$.

The expressions in (1.1) and (1.3) share a common 'multiplicative' structure. Guided by this analogy, we want to merge the two models together. To this end, we make the following elementary observation: if we block the server in an $\mathrm{M} / \mathrm{M} / 1$ queue then the number of waiting customers after $n$ arrivals is $A_{n}=n$. Also, $\left(A_{n}\right)_{n}$ can be viewed as the (not so random) random walk on the pair $((\mathbb{N},+),\{1\})$ associated with the probability $v: v(1)=1$. Now, we replace the trivial random walk $\left(A_{n}\right)_{n}$ by another, more complex, random walk $\left(X_{n}\right)_{n}$ on a plain triple $(X, \Sigma, v)$. Hence, the random walk $\left(X_{n}\right)_{n}$ constitutes the buffering mechanism in a queue with a blocked server. A zero-automatic queue is the model obtained when unblocking the server. The set $\Sigma$ is the set of possible classes for customers. Customers arrive at a constant rate $\lambda$. Upon arrival, a new customer (class $b$ ) interacts with the customer presently at the back-end of the buffer (class $a$ ), according to (1.2). At the front-end of the buffer, customers are served at a constant rate $\mu$. 
Let us comment on the name zero-automatic. Plain groups (see (2.1), below), are automatic in the sense of Epstein et al. [12]. Automatic groups form an important class of groups extensively studied in geometric group theory, the adjective 'automatic' referring to the existence of automata to recognize and multiply elements of the group. Now the pairs $(X, \Sigma)$ formed by a plain group with natural generators satisfy the zero-fellow traveller property (see [12] ). It was proposed in [22] to call $(X, \Sigma)$ a zero-automatic pair. By extension, a queue built upon $(X, \Sigma)$ is zero-automatic. The name is also supposed to evoke the local aspect of the interactions between customers in the buffer; see (1.2).

Let $\hat{\gamma}$ be the drift or rate of escape to infinity of the random walk $\left(X_{n}\right)_{n}$. We prove in Section 4 that the stability condition for the zero-automatic queue associated with $\left(X_{n}\right)_{n}$ is $\lambda \hat{\gamma}<\mu$. Under this condition, we prove in Section 5 that the stationary distribution $p$ for the queue-content process has a 'multiplicative' structure, given by

$$
p(u)=(1-\rho) \rho^{n} q\left(u_{1}\right) \cdots q\left(u_{n-1}\right) r\left(u_{n}\right) \quad \text { for all } u=u_{1} \cdots u_{n} \in L(X, \Sigma),
$$

for some numbers $\rho \in(0,1)$ and, for all $a \in \Sigma, q(a) \in(0,1)$ and $r(a) \in(0,1)$. (These numbers are, in general, different from their counterparts in (1.1) and (1.3).) Furthermore, the departure process from the queue is a Poisson process of rate $\rho \mu$. Thus, we have an analog of Burke's theorem for all zero-automatic queues. Using standard terminology, zero-automatic queues are quasi-reversible. To be more precise, given $\left(X_{n}\right)_{n}$, several variants of zero-automatic queues can be defined depending on the way in which the customers are incorporated into an empty queue (boundary condition). There is precisely one choice for which the result in (1.4) holds. The values of $\rho, q(\cdot)$, and $r(\cdot)$, as well as the right boundary condition, are obtained implicitly via the unique solution of a set of algebraic equations; see Theorems 5.1 and 5.2, below, for a precise statement.

Aside from the free monoid, the next simplest example of a plain monoid is the free group over one generator: $(\mathbb{Z},+)$. The zero-automatic queues associated with $(\mathbb{Z},\{-1,1\})$ are variations of Gelenbe's G-queues, or queues with positive and negative customers, which were extensively studied in the 1990s; see [13], [14], and the bibliography in [15]. General zero-automatic queues can be viewed as a wide generalization of this setting. Indeed, in a zero-automatic queue, different types of tasks (customers) can be modeled. Let us detail four of them which form a representative sample without exhausting all the types within the realm of zero-automaticity.

- Classical type. Tasks are processed one by one with no simplification occurring in the buffer; $a a=a a$. The corresponding pair is $(\mathbb{N},\{1\}) \sim\left(\{a\}^{*},\{a\}\right)$.

- Positive/negative type. Tasks are either positive $(a)$ or negative $\left(a^{-1}\right)$ and two consecutive tasks of opposite signs cancel each other; $a a^{-1}=a^{-1} a=1$. The corresponding pair is $(\mathbb{Z},\{1,-1\}) \sim\left(F(a),\left\{a, a^{-1}\right\}\right)$. The relevant applications for this type of task are discussed in [15].

- 'One equals many' type. It takes the same time to process one or several consecutive instances of the same task; $a a=a$. For instance, consider a ticket reservation where the number of requests is reflected only by an integer value in a menu-bar choice. The corresponding pair is $(\mathbb{B},\{a\})$ where $\mathbb{B}$ is the Boolean monoid $\mathbb{B}=\left\langle a \mid a^{2}=a\right\rangle$.

- 'Dating agency'type. Two instances of the same task cancel each other; $a a=1$. Consider a tennis player looking for a partner (to be provided by the server); when two such tasks are next to each other in the buffer, they leave to play a game instead of waiting in line. The corresponding pair is $\left(\mathbb{Z} / 2 \mathbb{Z}=\left\langle a \mid a^{2}=1\right\rangle,\{a\}\right)$. Instead of tennis players, we may 
consider music trio players, bridge players, etc., the corresponding group being $\mathbb{Z} / 3 \mathbb{Z}$, $\mathbb{Z} / 4 \mathbb{Z}$, etc.

To model a server where several of the above types (and possibly several copies of the same type) can be processed, we have just to perform the free product of the corresponding monoids or groups (see Section 2.1 for the definition).

The M/M/1 queue is the basic primitive for building Jackson networks, which have the remarkable property of having a 'product-form' stationary distribution. More generally, networks made of quasi-reversible nodes tend to have a product form distribution; see, for instance, [26]. In a subsequent work [9], we proved that this is indeed the case for both Jackson-type and Kelly-type networks of zero-automatic queues.

A preliminary version, without proofs, of the present paper has appeared in the conference proceedings [8].

\section{Preliminaries}

Notation. We denote by $\mathbb{Z}, \mathbb{N}$ and $\mathbb{R}_{+}$the integer, nonnegative integer, and real numbers, respectively. We denote by $\mathbb{N}^{*}$ and $\mathbb{R}_{+}^{*}$ the positive integer and real numbers, respectively. The symbol 'ப' is used for the disjoint union of sets. Given a set $T$ and $S \subset T$, we define $\mathbf{1}_{S}: T \rightarrow\{0,1\}$ by $\mathbf{1}_{S}(u)=1$ if $u \in S$ and $\mathbf{1}_{S}(u)=0$ otherwise. Given a set $T$, a vector $x \in \mathbb{R}^{T}$, and $S \subset T$, set $x(S)=\sum_{u \in S} x(u)$.

Let us recall the material needed for random walks on plain monoids. The presentation follows [22] and [23].

\subsection{Monoids and groups}

Given a set $\Sigma$, the free monoid generated by $\Sigma$ is denoted by $\Sigma^{*}$. The unit element is denoted by 1 or $1_{\Sigma^{*}}$. As usual, the elements of $\Sigma$ and $\Sigma^{*}$ are called letters and words, respectively. The subsets of $\Sigma^{*}$ are called languages. The length (number of letters) of a word $u$ is denoted by $|u|_{\Sigma}$. Let $(X, *)$ be a group or monoid with a set of generators $\Sigma$. The unit element of $X$ is denoted by $1_{X}$. When $X$ is a group, the inverse of $x \in X$ is denoted by $x^{-1}$. We always assume that $1_{X} \notin \Sigma$ and, in the group case, that $x \in \Sigma$ implies that $x^{-1} \in \Sigma$. The length with respect to $\Sigma$ of an element $x$ of $X$ is given by

$$
|x|_{\Sigma}=\min \left\{k \mid x=a_{1} * \cdots * a_{k}, a_{i} \in \Sigma\right\} .
$$

The Cayley graph $\mathcal{X}(X, \Sigma)$ of $X$ with respect to $\Sigma$ is the directed graph with nodes $X$ and $\operatorname{arcs}$ $u \rightarrow v$ if there exists an $a \in \Sigma$ such that $u * a=v$.

Consider a relation $R \subset \Sigma^{*} \times \Sigma^{*}$, and let ' $\sim_{R}$ ' be the least congruence on $\Sigma^{*}$ such that $u \sim_{R} v$ if $(u, v) \in R$. Let $X$ be isomorphic to the quotient monoid $\left(\Sigma^{*} / \sim_{R}\right)$. We say that $\langle\Sigma \mid u=v,(u, v) \in R\rangle$ is a monoid presentation of $X$ and we write $X=\langle\Sigma| u=v$, $(u, v) \in R\rangle$. Given a set $S$, we denote by $F(S)$ the free group generated (as a group) by $S$. Let $S^{-1}$ be the set of inverses of the generators. A monoid presentation of $F(S)$ is

$$
\left.F(S)=\left\langle S \sqcup S^{-1}\right| a a^{-1}=1, a^{-1} a=1, \text { for all } a \in S\right\rangle .
$$

Given two groups or monoids $X_{1}$ and $X_{2}$, we denote by $X_{1} \star X_{2}$ the free product of $X_{1}$ and $X_{2}$. Roughly, the elements of $X_{1} \star X_{2}$ are the finite alternate sequences of elements of $X_{1} \backslash\left\{1_{X_{1}}\right\}$ and $X_{2} \backslash\left\{1_{X_{2}}\right\}$, and the law is the concatenation with simplification. More rigorously, the definition is as follows. Let $S=X_{1} \sqcup X_{2}$. The free product, $X_{1} \star X_{2}$, is defined by the following monoid 
(a)

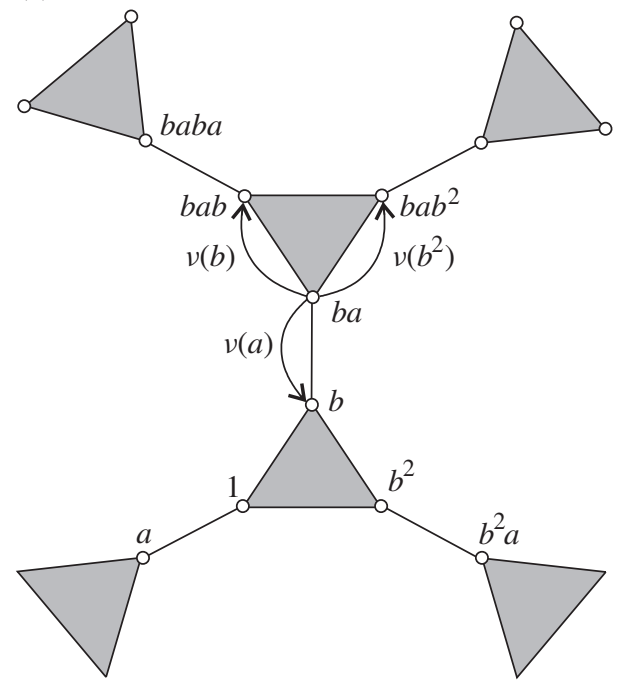

(b)
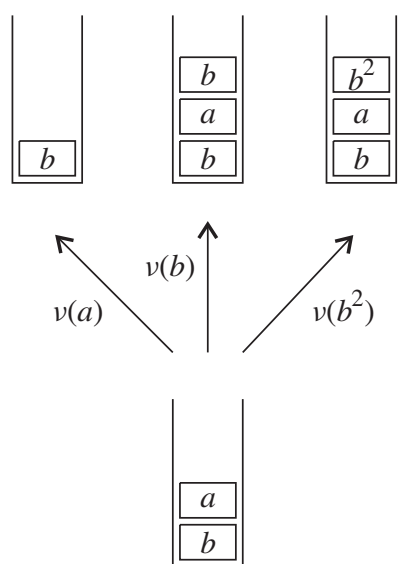

FIGURE 2: The random walk $(\mathbb{Z} / 2 \mathbb{Z} \star \mathbb{Z} / 3 \mathbb{Z}, v)$.

presentation:

$\langle S|$ (for all $u, v \in S^{*}$, for all $\left.i \in\{1,2\}\right), u 1_{X_{i}} v=u v$, (for all $a, b, c \in X_{i}$ such that $c=a * b$ ), uabv $\left.=u c v\right\rangle$.

If $X_{1}$ and $X_{2}$ are groups, then $X_{1} \star X_{2}$ is also a group. The free product of more than two groups or monoids is defined analogously. The Cayley graph of the group $\mathbb{Z} / 2 \mathbb{Z} \star \mathbb{Z} / 3 \mathbb{Z}$ is represented in Figure 2(a).

\subsection{Plain monoids and groups}

A plain monoid is a monoid $X$ of the form

$$
X=S^{*} \star F(T) \star X_{1} \star \cdots \star X_{k},
$$

where $S$ and $T$ are finite sets and $X_{1}, \ldots, X_{k}$ are finite monoids. A plain group is a plain monoid which is also a group. A plain monoid $X$ defined as in (2.1) is a plain group if and only if $S=\varnothing$ and $X_{1}, \ldots, X_{k}$ are groups. Define

$$
\Sigma=S \sqcup T \sqcup T^{-1} \sqcup X_{1} \backslash\left\{1_{X_{1}}\right\} \sqcup \cdots \sqcup X_{k} \backslash\left\{1_{X_{k}}\right\} .
$$

The set $\Sigma$ is a finite set of generators of $X$, which we call natural generators. Define the language $L(X, \Sigma) \subset \Sigma^{*}$ by

$$
L(X, \Sigma)=\left\{u_{1} \cdots u_{k} \mid \text { for all } i<k, u_{i} * u_{i+1} \notin \Sigma \cup\left\{1_{X}\right\}\right\} .
$$

It is easily seen that the set $L(X, \Sigma)$ is in bijection with the group elements. Below we often identify $X$ and $L(X, \Sigma)$. The following is a consequence of the definition of a plain monoid:

$$
a * b \in \Sigma \cup\left\{1_{X}\right\} \Longleftrightarrow b * a \in \Sigma \cup\left\{1_{X}\right\}, \quad a, b \in \Sigma
$$


To see that (2.3) holds, it is sufficient to check it case by case. It is convenient to introduce the sets

$$
\operatorname{Next}(a)=\left\{b \in \Sigma \mid b * a \notin \Sigma \cup\left\{1_{X}\right\}\right\}=\left\{b \in \Sigma \mid a * b \notin \Sigma \cup\left\{1_{X}\right\}\right\} \quad \text { for all } a \in \Sigma .
$$

Observe that

$$
L(X, \Sigma)=\left\{u_{1} \cdots u_{k} \mid \text { for all } i, u_{i-1} \in \operatorname{Next}\left(u_{i}\right)\right\}=\left\{u_{1} \cdots u_{k} \mid \text { for all } i, u_{i+1} \in \operatorname{Next}\left(u_{i}\right)\right\} .
$$

The following property, to be used later on, is also a direct consequence of the definition of a plain monoid:

$$
a * b \in \Sigma \Longrightarrow \operatorname{Next}(a)=\operatorname{Next}(b)=\operatorname{Next}(a * b) .
$$

Consider the directed graph of successors $(\Sigma, \rightarrow)$, where

$$
a \rightarrow b \quad \text { if } b \in \operatorname{Next}(a)
$$

Except for the case in which $X=F(T)$ where $|T|=1$, the graph $(\Sigma, \rightarrow)$ is strongly connected.

\subsection{Random walks on monoids and groups}

Let $(X, *)$ be a group or monoid with a finite set of generators $\Sigma$. Let $v$ be a probability distribution over $\Sigma$. Consider the Markov chain on the state space $X$ with one-step transition probabilities given by $\mathrm{P}_{x, x * a}=v(a)$ for all $x \in X$ and $a \in \Sigma$. This Markov chain is called the (right) random walk (associated with) $(X, v)$. Let $\left(x_{n}\right)_{n}$ be a sequence of i.i.d. RVs distributed according to $v$. Set

$$
X_{0}=1_{X}, \quad X_{n+1}=X_{n} * x_{n}=x_{0} * \cdots * x_{n} .
$$

Then the sequence $\left(X_{n}\right)_{n}$ is a realization of the random walk $(X, v)$. For all $x, y \in X$, we have $|x * y|_{\Sigma} \leq|x|_{\Sigma}+|y|_{\Sigma}$. Applying Kingman's subadditive ergodic theorem [18] yields the following (first noticed by Guivarc'h [16]): there exists a $\gamma \in \mathbb{R}_{+}$such that

$$
\lim _{n \rightarrow \infty} \frac{\left|X_{n}\right| \Sigma}{n}=\gamma \quad \text { almost surely (a.s.) and in } L^{p},
$$

for all $1 \leq p<\infty$. We call $\gamma$ the drift of the random walk. To illustrate this, consider the plain group $X=\mathbb{Z} / 2 \mathbb{Z} \star \mathbb{Z} / 3 \mathbb{Z}=\left\langle a \mid a^{2}=1\right\rangle \star\left\langle b \mid b^{3}=1\right\rangle$ and the natural generators $\Sigma=\left\{a, b, b^{2}=b^{-1}\right\}$. Let $v$ be a probability measure on $\Sigma$. Figure 2(a) represents a finite part of the infinite Cayley graph $\mathcal{X}(X, \Sigma)$, and the one-step transitions of the random walk $(X, v)$ starting from the state $b a$. Figure 2(b) shows the same one-step transitions of the group elements viewed as words of $L(X, \Sigma)$ (written from bottom to top).

\subsection{Random walks on plain monoids and groups}

It is convenient to introduce the notion of a plain triple.

Definition 2.1. A triple $(X, \Sigma, v)$ is plain if

(i) $X$ is an infinite plain monoid not isomorphic to $\mathbb{Z}$ or $\mathbb{Z} / 2 \mathbb{Z} \star \mathbb{Z} / 2 \mathbb{Z}$;

(ii) $\Sigma$ is a set of natural generators;

(iii) $v$ is a probability measure whose support is included in $\Sigma$ and generates $X$. 
Proposition 2.1. If $(X, \Sigma, v)$ is a plain triple, then the random walk $(X, v)$ is transient.

If $X$ is an infinite plain monoid with the support of $v$ generating $X$, there are only two cases in which $(X, v)$ is not transient

(1) the triple $\left(\mathbb{Z},\{-1,1\},\left\{\frac{1}{2}, \frac{1}{2}\right\}\right)$;

(2) the triples $(\mathbb{Z} / 2 \mathbb{Z} \star \mathbb{Z} / 2 \mathbb{Z},\{a, b\}, v)$, for any $\nu$, where $a$ and $b$ are the respective generators of the two cyclic groups.

As $\mathbb{Z}$ and $\mathbb{Z} / 2 \mathbb{Z} \star \mathbb{Z} / 2 \mathbb{Z}$ have been excluded from consideration, then the random walk $(X, v)$ is transient; see [22] for details. The case in which $X=\mathbb{Z}$ is specific. Some of the results below remain true but not all of them. For simplicity, we treat this case separately in Section 6.

Define

$$
\begin{aligned}
& \mathscr{B}=\left\{x \in \mathbb{R}^{\Sigma} \mid \text { for all } i, x(i)>0, \sum_{i} x(i)=1\right\}, \\
& \bar{B}=\left\{x \in \mathbb{R}^{\Sigma} \mid \text { for all } i, x(i) \geq 0, \sum_{i} x(i)=1\right\} .
\end{aligned}
$$

The traffic equations play an essential role in the study of the random walk $(X, v)$.

Definition 2.2. The Traffic Equations (TE) associated with a plain triple $(X, \Sigma, v)$ are the equations of the variables $(x(a))_{a \in \Sigma} \in \mathbb{R}_{+}^{\Sigma}$ defined by

$$
x(a)=v(a) x(\operatorname{Next}(a))+\sum_{b * d=a} v(b) x(d)+\sum_{\substack{d \in \operatorname{Next}(a) \\ b * d=1_{X}}} v(b) \frac{x(d)}{x(\operatorname{Next}(d))} x(a) \quad \text { for all } a \in \Sigma .
$$

An admissible solution of (2.7) is a solution belonging to $\mathscr{B}$.

By multiplying both sides of (2.7) by $\prod_{b} x(\operatorname{Next}(b))$, we obtain a new set of equations without denominators. With some abuse, a solution $r$ in $\overline{\mathcal{B}}$ of this set of equations is still called a solution of the TE.

The following proposition can be deduced easily from the proof of Theorem 4.5 of [22].

Proposition 2.2. Let $(X, \Sigma, v)$ be a plain triple. The traffic equations have a unique admissible solution.

The harmonic measure and the drift can be expressed as a function of the solution to the TE, which makes Proposition 2.2 interesting. Define the set $L^{\infty} \subset \Sigma^{\mathbb{N}}$ by

$$
L^{\infty}=\left\{u_{0} u_{1} \cdots u_{k} \cdots \in \Sigma^{\mathbb{N}} \mid \text { for all } i \in \mathbb{N}, u_{i+1} \in \operatorname{Next}\left(u_{i}\right)\right\} .
$$

A word belongs to $L^{\infty}$ if and only if all its finite prefixes belong to $L(X, \Sigma)$. The set $L^{\infty}$ should be viewed as the 'boundary' of $X$.

Let $\left(X_{n}\right)_{n}$ be a realization of the random walk which is transient by Proposition 2.1. The harmonic measure of the random walk is the probability measure $v^{\infty}$ on $L^{\infty}$ with finitedimensional marginals defined by

$$
v^{\infty}\left(u_{1} \cdots u_{k} \Sigma^{\mathbb{N}}\right)=\mathrm{P}\left\{\text { there exists an } N, \text { for all } n \geq N, X_{n} \in u_{1} \cdots u_{k} \Sigma^{*}\right\},
$$


for all $u_{1} \cdots u_{k} \in L(X, \Sigma)$. This indeed defines a measure on $L^{\infty}$ because the random walk is transient, and because $X_{n}$ and $X_{n+1}$ differ by at most their last symbol. Intuitively, the harmonic measure $v^{\infty}$ gives the direction in which $\left(X_{n}\right)_{n}$ goes to infinity.

For a proof of the following theorem the reader is referred to the proofs of Theorem 4.5 of [22] and Theorem 3.3 of [23]. In the specific case of the free group, the proof appears in [11], [25], and also the survey [21].

Theorem 2.1. Let $(X, \Sigma, v)$ be a plain triple. Let $\hat{r}=(\hat{r}(a))_{a \in \Sigma}$ be the unique admissible solution to the traffic equations. Set $\hat{q}(a)=\hat{r}(a) / \hat{r}(\operatorname{Next}(a))$, for all $a \in \Sigma$. The harmonic measure $v^{\infty}$ of the random walk $(X, v)$ is given by

$$
v^{\infty}\left(u_{1} \cdots u_{k} \Sigma^{\mathbb{N}}\right)=\hat{q}\left(u_{1}\right) \cdots \hat{q}\left(u_{k-1}\right) \hat{r}\left(u_{k}\right) \text { for all } u_{1} \cdots u_{k} .
$$

The drift of the random walk is given by

$$
\hat{\gamma}=\sum_{a \in \Sigma} v(a)\left[\hat{r}(\operatorname{Next}(a))-\sum_{b \mid a * b=1_{X}} \hat{r}(b)\right] .
$$

\section{The zero-automatic queue}

We first define the zero-automatic queue informally, before defining it formally in Definition 3.1. Let $X$ be a plain monoid, $\Sigma$ be a set of natural generators, and $v$ a probability measure on $\Sigma$. The associated zero-automatic queue is formed by a simple single server queue with FIFO discipline and an infinite capacity buffer in which the buffering occurs according to the random walk $(X, v)$. It is a multiclass queue (classes $\Sigma$ ) but the class does not influence the way customers get served, only the way they get buffered. More precisely, the instants of customer arrivals are given by a Poisson process of rate $\lambda$, and each customer carries a mark, or class, which is an element of $\Sigma$. The sequence of marks is i.i.d. of law $v$. Upon arrival, a new customer interacts with the customer presently at the back-end of the buffer and, depending on their respective classes, say $b$ and $a$, one of three following possible events occurs:

(i) if $b * a=1_{X}$, then the two customers leave the queue;

(ii) if $b * a=c \in \Sigma$, then the two customers merge to create a customer of type $c$;

(iii) otherwise, customer $b$ takes place at the back-end of the buffer, behind customer $a$.

In the mean time, at the front-end of the buffer, the customers are served one by one and at a constant rate $\mu$ by the server. To be complete, we need to specify how customers are incorporated when the buffer is empty. Several variants may be considered, and we view this 'boundary condition' as an additional parameter of the model. The resulting flexibility in the definition of a zero-automatic queue will turn out to be a crucial point.

According to the above description, the queue-content (the sequence of classes of customers in the buffer) is a continuous-time jump Markov process. The more formal definition of the queue is given via the infinitesimal generator of this process.

Definition 3.1. (Zero-automatic queue.) Consider a plain triple $(X, \Sigma, v)$. Let $L(X, \Sigma)$ be the set of words defined in (2.2). Consider $r \in \overline{\mathcal{B}}$ (see (2.6)) and $\lambda, \mu \in \mathbb{R}_{+}^{*}$. The zero-automatic queue of type $(X, \Sigma, v, r, \lambda, \mu)$ is defined as follows. The queue-content $(M(t))_{t \in \mathbb{R}_{+}}$is a continuous-time jump Markov process on the state space $L(X, \Sigma)$ with infinitesimal generator 
$Q$ defined by

$$
\begin{aligned}
& Q(u, b u)=\lambda v(b) \quad \text { for all } b \in \operatorname{Next}\left(u_{n}\right), \\
& Q\left(u, c u_{n-1} \cdots u_{1}\right)=\lambda \sum_{b \mid b * u_{n}=c} v(b) \text { for all } c \in \Sigma \backslash\left\{u_{n}\right\} \text { such that there exists } b \in \Sigma, \\
& b * u_{n}=c, \\
& Q\left(u, u_{n-1} \cdots u_{1}\right)=\lambda \sum_{b \mid b * u_{n}=1_{X}} v(b), \\
& Q\left(u, u_{n} \cdots u_{2}\right)=\mu,
\end{aligned}
$$

for all $u=u_{n} \cdots u_{1} \in L(X, \Sigma) \backslash \cup_{a \in \Sigma}\{a\}^{*}$. Furthermore, for all $a \in \Sigma$ such that $a \in \operatorname{Next}(a)$ and for all $n \geq 1$,

$$
\begin{aligned}
& Q\left(a^{n}, b a^{n}\right)=\lambda v(b) \quad \text { for all } b \in \operatorname{Next}(a), \\
& \begin{array}{l}
Q\left(a^{n}, c a^{n-1}\right)=\lambda \sum_{b \mid b * a=c} v(b) \quad \text { for all } c \in \Sigma \backslash\{a\} \text { such that there exists } b \in \Sigma, \\
b * a=c,
\end{array} \\
& Q\left(a^{n}, a^{n-1}\right)=\mu+\lambda \sum_{b \mid b * a=1_{X}} v(b),
\end{aligned}
$$

and, finally, the boundary condition is

$$
Q\left(1_{\Sigma^{*}}, a\right)=\lambda \nu(a) r(\operatorname{Next}(a)) \quad \text { for all } a \in \Sigma .
$$

We denote by $\mathrm{M} / \mathrm{M} /(X, \Sigma)$ any zero-automatic queue of type $(X, \Sigma, v, r, \lambda, \mu)$.

Remark 3.1. The reason behind choosing the boundary condition to be of the form given in (3.4) is as follows: the buffer-content is viewed as the visible part of an iceberg consisting of an infinite word of $L^{\infty}$; see (2.8). When the buffer is empty, new customers are incorporated depending on the invisible part of the iceberg, whose first marginal is assumed to be $r$. This last point will be justified a posteriori in Theorem 5.1.

The simplest example of a zero-automatic queue is the one associated with the free monoid $(\mathbb{N},+)$. The triple $(\mathbb{Z},\{-1,1\}, v)$, where $v$ is a probability measure on $\{1,-1\}$, is not plain. However, it is simple and interesting to generalize Definition 3.1 in order to define a zeroautomatic queue associated with the free group $(\mathbb{Z},+)$. We now discuss the zero-automatic queues associated with $(\mathbb{N},+)$ and $(\mathbb{Z},+)$.

For the simple queue, consider the free monoid $X=\{a\}^{*}=\left\{a^{k}, k \in \mathbb{N}\right\}$ over the single generator set $\Sigma=\{a\}$. Hence, for any $\lambda, \mu \in \mathbb{R}_{+}^{*}$, there is only one possible associated queue, $(X, \Sigma, \nu, r, \lambda, \mu)$, where $v(a)=r(a)=1$. By specializing the infinitesimal generator $Q$ given in Definition 3.1, we obtain

$$
Q\left(a^{n}, a^{n+1}\right)=\lambda, \quad Q\left(a^{n+1}, a^{n}\right)=\mu, \quad \text { for all } n \in \mathbb{N} .
$$

This is the simple M/M/1/ $\infty$ FIFO queue with an arrival rate $\lambda$ and a service rate $\mu$.

For the G-queue, consider the free group $X=F(a)=\left\{a^{k}, k \in \mathbb{Z}\right\}$ and the set of generators $\Sigma=\left\{a, a^{-1}\right\}$. Let $v$ be a probability measure on $\Sigma$ such that $v(a)>0$ and $v\left(a^{-1}\right)>0$. 
Consider $r \in \mathcal{B}$ and $\lambda, \mu \in \mathbb{R}_{+}^{*}$. The zero-automatic queue $(F(a), \Sigma, \nu, r, \lambda, \mu)$ has an infinitesimal generator $Q$ given by

$$
\begin{array}{ll}
Q\left(a^{n}, a^{n+1}\right)=\lambda \nu(a), & Q\left(a^{n+1}, a^{n}\right)=\mu+\lambda \nu\left(a^{-1}\right), \\
Q\left(a^{-n}, a^{-(n+1)}\right)=\lambda \nu\left(a^{-1}\right), & Q\left(a^{-(n+1)}, a^{-n}\right)=\mu+\lambda \nu(a), \\
Q\left(1_{\Sigma^{*}}, a\right)=\lambda \nu(a) r(a), & Q\left(1_{\Sigma^{*}}, a^{-1}\right)=\lambda \nu\left(a^{-1}\right) r\left(a^{-1}\right),
\end{array}
$$

for all $n \in \mathbb{N}$. This is close to the mechanism of the G-queue; a queue with positive and negative customers introduced by Gelenbe [14], [15]. With respect to the G-queue, one originality of the $\mathrm{M} / \mathrm{M} /(F(a), \Sigma)$ queue is that negative and positive customers play symmetrical roles. Another one is the treatment of the boundary condition.

As the triple $\left(F(a),\left\{a^{-1}, a\right\}, v\right)$ is not plain according to Definition 2.1, the above queue is not covered by the results of Sections 4 and 5. However, part of the results remain true, and we come back specifically to this model in Section 6.1 and Section 6.2.4.

As an extension, it is possible to generalize Definition 3.1 in order to define a zero-automatic queue of type $\mathrm{GI} / \mathrm{GI} /(X, \Sigma)$ and $\mathrm{G} / \mathrm{G} /(X, \Sigma)$. Roughly, the description would go as follows. The buffering mechanism is kept unchanged; the sequence of interarrival times and classes of customers is i.i.d. (stationary and ergodic, respectively); the sequence of service times at the server is i.i.d. (stationary and ergodic, respectively) and independent of the arrivals.

\subsection{Comparison with other models in the literature}

Under stability condition, we will see that a zero-automatic queue has the 'Poisson output' property. Also, a zero-automatic queue is 'quasi-reversible', at least in the sense of Chao et al. [6, Definition 3.4]. There exist many examples of queues with such properties; see for instance Kelly [17] or Chao et al. [6]. However, zero-automatic queues are quite different from the existing models. Let us detail the comparison with the model of Chao et al. [6] (see also [5]). Their model is a wide generalization of Gelenbe's G-queue with signals, batch arrivals, and batch departures. In a sense, zero-automatic queues can also be viewed as a wide generalization of G-queues. Other common features between the models include: nonlinear traffic equations, an output rate different from the input rate, and subtle boundary conditions to obtain a product form. Despite these similarities, the models are quite orthogonal. A main advantage of zeroautomatic queues is the possibility that two customers can merge and create a new customer of a different type. The algebraic foundation of zero-automatic queues is another originality.

It is also worthwhile comparing the zero-automatic queue with another model for queues introduced by Yeung and Sengupta [28] (referred to as the YS model in the following); see also He [19]. A common feature between the two models is the structure of the state space: a tree for the YS model (or the cartesian product of a tree and a finite set), and a more general treelike graph for the zero-automatic queue. In particular, both models correspond to multiclass queues, and the buffer content is coded by a word over the alphabet of classes. A second common feature is that the effect of a new arrival is either to add, to modify the class of, or to remove a customer at the back-end of the buffer (in the YS model, the removal/modification may affect several customers at the back-end of the buffer). The first central difference between the two models is that departures occur at the front-end of the buffer in the zero-automatic queue and at the back-end in the YS model. Therefore, the former is a FIFO queue while the latter is a last-in-first-out (LIFO) queue. We have illustrated the FIFO mechanism of the zero-automatic queue in Figure 3. The second important difference between the two models concerns the type of results which are proved. In a stable zero-automatic queue the buffer content has 

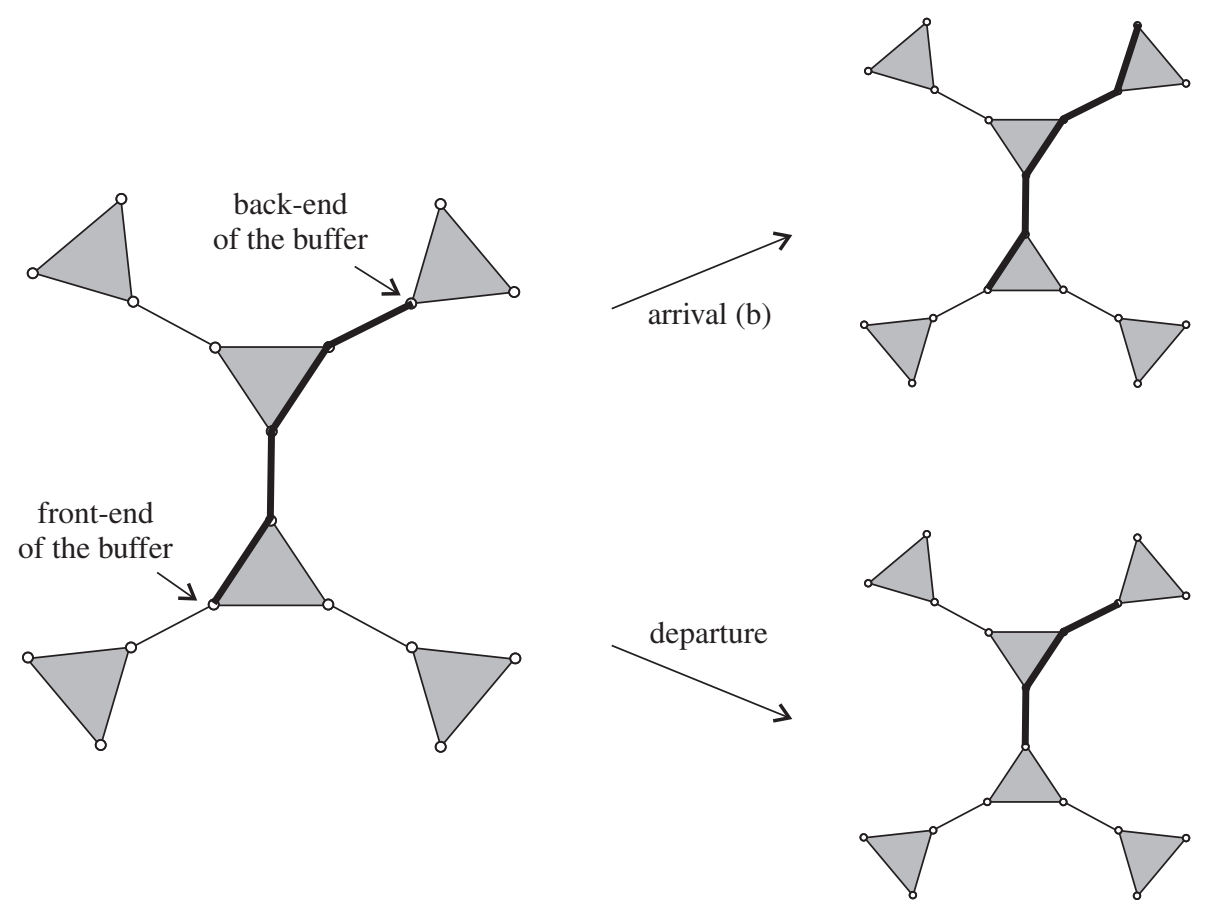

FIGURE 3: The effect of an arrival and a departure on the content of the buffer in a zero-automatic queue built on the group $\mathbb{Z} / 2 \mathbb{Z} \star \mathbb{Z} / 3 \mathbb{Z}$.

a 'product form' stationary distribution (see Theorem 5.1); whereas, in the YS model it has only a 'matrix product form' stationary distribution (see [28, Section 2]) In the YS model, obtaining the stronger 'product form' stationary distribution requires additional assumptions; see [28, Section 6]. To conclude the comparison, the original flavor of the zero-automatic queue comes from the underlying group or monoid structure. It is this algebraic foundation which accounts for the ability to obtain the strong product form results.

\section{Stability condition for a zero-automatic queue}

Throughout Sections 4 and 5, we define the model as follows. Let $(X, \Sigma, v)$ be a plain triple. Fix $\lambda$ and $\mu$ in $\mathbb{R}_{+}^{*}$ and $r$ in $\mathscr{B}$. Consider the zero-automatic queue $(X, \Sigma, \nu, r, \lambda, \mu)$.

Let $M=\left(M_{t}\right)_{t}$ be the queue-content process, and $Q$ the infinitesimal generator. Lemma 4.1 is a direct consequence of the strong connectivity of the graph $(\Sigma, \rightarrow)$ defined in (2.4).

Lemma 4.1. The process $M$ is irreducible.

The aim of this section is to prove Proposition 4.1, which characterizes the stability region of the zero-automatic queue.

Proposition 4.1. Let $\hat{\gamma}$ be the drift of the random walk $(X, v)$. We have

$$
\begin{aligned}
& {[\lambda \hat{\gamma}<\mu] \Longleftrightarrow M \text { is ergodic, }} \\
& {[\lambda \hat{\gamma}=\mu] \Longleftrightarrow M \text { is null recurrent, }} \\
& {[\lambda \hat{\gamma}>\mu] \Longleftrightarrow M \text { is transient. }}
\end{aligned}
$$


Consider an excursion of $M$ from the instant $t=0$ at which it is assumed to leave state $1_{\Sigma^{*}}$, to the instant $R_{M}$ which corresponds to the first return to state $1_{\Sigma^{*}}$. Recall that $M$ is transient if and only if $\mathrm{P}\left\{R_{M}=\infty\right\}>0$ and ergodic if and only if $\mathrm{E}\left[R_{M}\right]<\infty$. It is convenient to use the following representation for $M$. Let $A=\left(A_{0}=0, A_{1}, A_{2}, \ldots\right)$, where $\left(A_{1}, A_{2}, \ldots\right)$ are the time points of a time-stationary Poisson process of rate $\lambda$ on $\mathbb{R}_{+}$. Let $N_{A}=\left(N_{A}(t)\right)_{t}$ be the corresponding counting process and let $N_{D}=\left(N_{D}(t)\right)_{t}$ be the counting process of a time-stationary Poisson process of rate $\mu$ on $\mathbb{R}_{+}$. Let $\tilde{X}=\left(\tilde{X}_{n}\right)_{n}$ be a realization of the random walk $(X, v)$ viewed as evolving on $L(X, \Sigma)$; see (2.5). Assume that $A, \tilde{X}$, and $N_{D}$ are mutually independent. Let $\left(X_{t}\right)_{t}$ be the continuous-time jump Markov process on the state space $L(X, \Sigma)$ defined by

$$
X_{t}=\tilde{X}_{n+1} \quad \text { on }\left[A_{n}, A_{n+1}\right) .
$$

For all $t$ in the interval $\left[0, R_{M}\right)$, we have

$$
\left|M_{t}\right|_{\Sigma}=\left|X_{t}\right|_{\Sigma}-N_{D}(t)
$$

Here, $X_{t}$ is the queue-content at time $t$ if no service has been completed. Observe that the first letter of $X_{t}$ corresponds to the front-end of the buffer (the right-end in Figure 1), and the last letter to the back-end (the left-end in Figure 1).

The counting process of a Poisson process satisfies a strong law of large numbers. We obtain, a.s.,

$$
\lim _{t \rightarrow \infty} \frac{N_{A}(t)}{t}=\lambda, \quad \lim _{t \rightarrow \infty} \frac{N_{D}(t)}{t}=\mu .
$$

We also have, a.s.,

$$
\lim _{n \rightarrow \infty} \frac{\left|\tilde{X}_{n}\right|_{\Sigma}}{n}=\hat{\gamma}
$$

where $\hat{\gamma}$ is the drift of the random walk $(X, v)$. So we have, a.s.,

$$
\lim _{n \rightarrow \infty} \frac{\left|X_{t}\right|_{\Sigma}}{t}=\lim _{t \rightarrow \infty} \frac{\left|X_{t}\right|_{\Sigma}}{N_{A}(t)} \times \frac{N_{A}(t)}{t}=\lambda \hat{\gamma} .
$$

We can now prove the following result.

Lemma 4.2. If $\lambda \hat{\gamma}<\mu$ then $M$ is recurrent. If $\lambda \hat{\gamma}>\mu$ then $M$ is transient.

Proof. We show the first statement by contraposition. If $M$ is transient then $\mathrm{P}\left\{R_{M}=\infty\right\}>$ 0 . Using (4.1) and (4.2), we obtain that a.s. on the event $\left\{R_{M}=\infty\right\}$, we have

$$
\lim _{t \rightarrow \infty} \frac{\left|M_{t}\right|_{\Sigma}}{t}=\lambda \hat{\gamma}-\mu .
$$

To avoid a contradiction, we must have $\lambda \hat{\gamma}-\mu \geq 0$. Now assume that $\lambda \hat{\gamma}>\mu$. Using the independence of $\left(X_{t}\right)_{t}$, and $N_{D}$, and the regenerative properties of $R_{M}$, it is easily shown that $\mathrm{P}\left\{\right.$ for all $\left.t,\left|M_{t}\right|_{\Sigma}>0\right\}>0$. In particular, $\mathrm{P}\left\{R_{M}=\infty\right\}>0$ and $M$ is transient. This completes the proof.

To obtain the stronger results in Proposition 4.1, the idea is to approximate the zero-automatic queue by a simple queue with a Markov additive arrival process, and then to use standard results from queueing theory. 
As $\left(X_{t}\right)_{t}$ is transient (Proposition 2.1), there exists an a.s. finite $T_{0}$ such that, for all $t \geq T_{0}$, $\left|X_{t}\right|_{\Sigma} \geq 1$. For notational simplicity, we assume that $T_{0}=0$. Define the random variables

$$
T_{k}=\inf \left\{t \mid \text { for all } s \geq t,\left|X_{s}\right|_{\Sigma} \geq k+1\right\}, \quad \tau_{k}=T_{k+1}-T_{k},
$$

for all $k \geq 1$. The RVs, $T_{k}$, are a.s. finite because $\left(X_{t}\right)_{t}$ is transient, and we have $\left(T_{0}=0\right)<$ $T_{1}<T_{2}<\cdots$ a.s. By transience, $\lim _{t} X_{t}$ is a (random) infinite word on the alphabet $\Sigma$, let us write it as $\lim _{t} X_{t}=U_{0} U_{1} U_{2} \cdots$. By definition, see Section 2, the law of $U_{1} U_{2} \cdots$ is the harmonic measure $v^{\infty}$ of the random walk $(X, v)$. Observe that

$$
X_{T_{k}^{-}}=U_{0} \cdots U_{k-1} \quad \text { for all } t \geq T_{k}, X_{t}=U_{0} \cdots U_{k-1} Y_{t}, Y_{t} \in L(X, \Sigma) \backslash\left\{1_{\Sigma^{*}}\right\} .
$$

According to Theorem 2.1, we have

$$
\begin{aligned}
\mathrm{P}\left\{U_{0} \cdots U_{k-1}=u_{0} \cdots u_{k-1}\right\} & =\frac{\hat{r}\left(u_{0}\right)}{\hat{r}\left(\operatorname{Next}\left(u_{0}\right)\right)} \cdots \frac{\hat{r}\left(u_{k-2}\right)}{\hat{r}\left(\operatorname{Next}\left(u_{k-2}\right)\right)} \hat{r}\left(u_{k-1}\right) \\
& =\hat{r}\left(u_{0}\right) \frac{\hat{r}\left(u_{1}\right)}{\hat{r}\left(\operatorname{Next}\left(u_{0}\right)\right)} \cdots \frac{\hat{r}\left(u_{k-1}\right)}{\hat{r}\left(\operatorname{Next}\left(u_{k-2}\right)\right)}
\end{aligned}
$$

for all $u_{0} \cdots u_{k-1} \in L(X, \Sigma)$. It follows that $\left(U_{k}\right)_{k}$ is a Markov chain with initial distribution $\hat{r}$ and transition matrix $P$ given by

$$
P_{a, b}= \begin{cases}\frac{\hat{r}(b)}{\hat{r}(\operatorname{Next}(a))} & \text { if } b \in \operatorname{Next}(a), \\ 0 & \text { otherwise }\end{cases}
$$

for all $a, b \in \Sigma$. The matrix $P$ is irreducible as a direct consequence of the strong connectivity of the graph of successors $(\Sigma, \rightarrow)$; see (2.4). Let $\pi$ be the stationary distribution of $P$ characterized by $\pi P=\pi$. In general, the Markov chain $\left(U_{k}\right)_{k}$ is not stationary, i.e. $\hat{r}$ is different from $\pi$. (See [23, Proposition 3.6] for a sufficient condition on $(X, v)$ ensuring that $\hat{r}=\pi$.)

Consider now the sequence $(U, \tau)=\left(\left(U_{k}, \tau_{k}\right)\right)_{k}$. A consequence of the above is that $(U, \tau)$ is a Markov chain with transition function depending only on the first coordinate. According to the classical terminology, the sequence $\left(T_{k}\right)_{k}$ is a Markov additive process (MAP).

Consider the simple queue of type MAP/M/1 with arrival process $\left(T_{k}\right)_{k}$, and a service process driven by $N$. Let $\left(\sigma_{k}\right)_{k}$ be the corresponding sequence of service times. We deduce from (4.2), a.s. and in $L^{1}$, that

$$
\lim _{n \rightarrow \infty} \frac{1}{n} \sum_{i=1}^{n}\left(\sigma_{i}-\tau_{i}\right)=\frac{1}{\mu}-\frac{1}{\lambda \hat{\gamma}} .
$$

Let $Z=\left(Z_{t}\right)_{t}$ be the queue-length process of this queue. Let $R_{Z}$ be the first instant of return to zero for the process $Z$. Applying standard results for MAP/GI/1 queues, see for instance [2, Proposition 4.2, Chapter X], we obtain

$$
\begin{aligned}
{[\lambda \hat{\gamma}<\mu] } & \Longrightarrow\left[\mathrm{E}\left[R_{Z}\right]<\infty\right], \\
{[\lambda \hat{\gamma}=\mu] } & \Longrightarrow\left[R_{Z} \text { a.s. finite, } \mathrm{E}\left[R_{Z}\right]=\infty\right] .
\end{aligned}
$$

Concentrating on the mechanism of the zero-automatic queue, it is not difficult to see that

$$
\left[T_{k} \leq R_{Z}<T_{k+1}\right] \Longrightarrow\left[T_{k} \leq R_{Z} \leq R_{M}<T_{k+1}\right]
$$


Hence, the queue MAP/M/1 is a good approximation of the zero-automatic queue. In particular, the two implications in (4.3)-(4.4) also hold for $R_{M}$. In view of Lemma 4.2, this completes the proof of Proposition 4.1.

Remark. The above proof does not rely in an essential way on the Markovian assumption. For instance, an analog of Proposition 4.1 can clearly be written for a zero-automatic queue of type $\mathrm{GI} / \mathrm{GI} /(X, \Sigma)$.

\section{Stationary distribution of a stable queue}

\subsection{The twisted traffic equations}

The traffic equations, see Definition 2.2, play a central role in studying the random walk. We now introduce equations which play a similar role for the queue.

Definition 5.1. (Twisted traffic equations.) The Twisted traffic equations (TTE) associated with $(X, \Sigma, \nu, \lambda, \mu)$ are the equations of the variables $(\eta, x)$, where $\eta \in \mathbb{R}_{+}^{*}$ and $x=$ $(x(a))_{a \in \Sigma} \in \mathbb{R}_{+}^{\Sigma}$, defined by

$$
\begin{aligned}
\eta(\lambda+\mu) x(a)= & \eta^{2} \mu x(a)+\lambda v(a) x(\operatorname{Next}(a))+\eta \lambda \sum_{b * d=a} v(b) x(d) \\
& +\eta^{2} \lambda \sum_{\substack{d \in \operatorname{Next}(a) \\
b * d=1_{X}}} v(b) \frac{x(d)}{x(\operatorname{Next}(d))} x(a) .
\end{aligned}
$$

To indicate the future role of the TTE we examine the case $(X, \Sigma)=\left(\{a\}^{*},\{a\}\right)$ considered at the end of Section 3. Recall that there is only one possible variant for the queue $\mathrm{M} / \mathrm{M} /\left(\{a\}^{*},\{a\}\right)$ which is equivalent to the simple $\mathrm{M} / \mathrm{M} / 1$ queue. By simplifying (5.1), we obtain

$$
\rho(\lambda+\mu)=\rho^{2} \mu+\lambda .
$$

Compare this with the global balance equations of the $\mathrm{M} / \mathrm{M} / 1$ queue, we obtain

$$
\pi(n)(\lambda+\mu)=\pi(n-1) \lambda+\pi(n+1) \mu .
$$

By substituting $\pi(n)=\pi(0) \rho^{n}$ in (5.3) and simplifying, we obtain (5.2).

According to Proposition 2.2, there is a unique admissible solution to the traffic equations, which we denote by $\hat{r}=(\hat{r}(a))_{a \in \Sigma}$. We denote by $\hat{\gamma}$ the drift of the random walk $(X, v)$. Consider $x \in \overline{\mathcal{B}}$. Define

$$
A(x)=\sum_{a \in \Sigma} v(a) \sum_{b \in \operatorname{Next}(a)} x(b), \quad B(x)=\sum_{a * b \in \Sigma} v(a) x(b), \quad C(x)=\sum_{a * b=1_{X}} v(a) x(b) .
$$

It is easy to check that

$$
A(x)+B(x)+C(x)=1 .
$$

This point was the crux of the argument in proving Proposition 2.4 in [22]. Observe that a simple rewriting of (2.9) gives

$$
\hat{\gamma}=A(\hat{r})-C(\hat{r}) \text {. }
$$

Let us investigate some properties of the solutions to the twisted traffic equations. First, if $(\rho, r)$ is a solution to the TTE with $r \in \bar{B}$, then $r$ belongs to $\mathcal{B}$. This follows directly from the 
shape of the TTE and from the strong connectivity of the graph $(\Sigma, \rightarrow)$; see (2.4). Second, if we set $\eta=1$ in the TTE (5.1) and perform the obvious simplifications, then we obtain the TE (2.7). This implies that $(1, \hat{r})$ is a solution to the TTE for all $\lambda$ and $\mu$.

Lemma 5.1. Let $(\rho, r), \rho \in \mathbb{R}_{+}^{*}$ and $r \in \mathscr{B}$, be a solution to the TTE. We have either $(\rho, r)=(1, \hat{r})$ or

$$
\rho=\frac{\lambda \sum_{a \in \Sigma} v(a) r(\operatorname{Next}(a))}{\mu+\lambda \sum_{a * b=1_{X}} v(a) r(b)}=\frac{\lambda A(r)}{\mu+\lambda C(r)} .
$$

Proof. By summing all the equations of (5.1), we obtain

$$
\begin{aligned}
\rho(\lambda+\mu)= & \rho^{2} \mu+\lambda \sum_{a \in \Sigma} v(a) r(\operatorname{Next}(a))+\rho \lambda \sum_{a * b \in \Sigma} v(a) r(b) \\
& +\rho^{2} \lambda \sum_{\substack{d \in \operatorname{Next}(a) \\
b * d=1_{X}}} v(b) \frac{r(d)}{r(\operatorname{Next}(d))} r(a) \\
= & \rho^{2} \mu+\lambda A(r)+\rho \lambda B(r)+\rho^{2} \lambda C(r) .
\end{aligned}
$$

Replacing $B(r)$ by $1-A(r)-C(r)$ in the above, we obtain

$$
(\rho-1)[(\mu+\lambda C(r)) \rho-\lambda A(r)]=0 .
$$

If $\rho=1$, we have seen that the TTE reduces to the TE which implies, by Proposition 2.2, that $(\rho, r)=(1, \hat{r})$. Otherwise, we must have $(\mu+\lambda C(r)) \rho-\lambda A(r)=0$. This completes the proof.

The relevant solutions to the TTE will turn out to be the ones satisfying (5.6). This leads us to the next definition.

Definition 5.2. A solution $(\rho, r)$ to the TTE is called an admissible solution if $\rho \in \mathbb{R}_{+}^{*}, r \in \mathscr{B}$, and if (5.6) is satisfied.

Lemma 5.2. If $\lambda \hat{\gamma}=\mu$, then $(1, \hat{r})$ is an admissible solution to the TTE. If $(1, r)$ is an admissible solution to the TTE, then $r=\hat{r}$ and $\lambda \hat{\gamma}=\mu$.

Proof. Assume that $\lambda \hat{\gamma}=\mu$. We know that $(1, \hat{r})$ is a solution to the TTE but we need to check that it is admissible. By definition, it is admissible if

$$
1=\frac{\lambda A(\hat{r})}{\mu+\lambda C(\hat{r})} \Longleftrightarrow \lambda(A(\hat{r})-C(\hat{r}))=\mu .
$$

We conclude by recalling that $\hat{\gamma}=A(\hat{r})-C(\hat{r})$; see (5.5). Assume now that $(1, r)$ is an admissible solution to the TTE. As $\rho=1$, the TTE reduces to the TE implying that $r=\hat{r}$. Now replacing $(\rho, r)$ by $(1, \hat{r})$ in (5.6) yields $\lambda \hat{\gamma}=\mu$. This completes the proof.

More generally, admissible solutions always exist.

Lemma 5.3. There exists an admissible solution to the TTE. 
Proof. Replace $\eta$ by $\lambda A(x) /(\mu+\lambda C(x))$ in (5.1). The resulting equations in $x$ can be viewed as a fixed point equation of the type $\Psi(x)=x$. The corresponding application $\Psi:\left(\mathbb{R}_{+}^{*}\right)^{\Sigma} \longrightarrow$ $\left(\mathbb{R}_{+}^{*}\right)^{\Sigma}$ has the following form:

$$
\begin{aligned}
\Psi(x)(a)=\frac{1}{\lambda+\mu}[ & \frac{\lambda A(x)}{\mu+\lambda C(x)}\left[\mu x(a)+\lambda \sum_{\substack{d \in \operatorname{Next}(a) \\
b * d=1_{X}}} v(b) \frac{x(d)}{x(\operatorname{Next}(d))} x(a)\right] \\
& \left.+\lambda \sum_{b * d=a} v(b) x(d)+\frac{\mu+\lambda C(x)}{A(x)} v(a) x(\operatorname{Next}(a))\right],
\end{aligned}
$$

for $a \in \Sigma$ and for $x \in\left(\mathbb{R}_{+}^{*}\right)^{\Sigma}$. Consider $x \in \mathscr{B}$. By summing the equations in (5.7) and using (5.4), we obtain

$$
\sum_{a \in \Sigma} \Psi(x)(a)=\frac{1}{\lambda+\mu}[\lambda A(x)+\lambda B(x)+\mu+\lambda C(x)]=1 .
$$

Thus, we have proved that $\Psi(\mathscr{B}) \subset \mathscr{B}$. The end of the proof follows very closely the proof of Theorem 4.5 of [22]. For the sake of completeness, we recall the argument. To use a fixed point theorem, we need to define $\Psi$ on a compact and convex set. The set $\overline{\mathcal{B}}$, which is the closure of $\mathscr{B}$, is a compact and convex subset of $\mathbb{R}^{\Sigma}$. But the map $\Psi$ cannot in general be extended continuously on $\overline{\mathscr{B}}$. More precisely, $\Psi(x), x \in \overline{\mathscr{B}} \backslash \mathscr{B}$, can be defined unambiguously if and only if $x(\operatorname{Next}(u)) \neq 0$ for all $u$. For $x \in \overline{\mathscr{B}} \backslash \mathscr{B}$, let $\Psi(x) \subset \overline{\mathscr{B}}$ be the set of possible limits of $\Psi\left(x_{n}\right), x_{n} \in \mathscr{B}, x_{n} \rightarrow x$. We have extended $\Psi$ to a correspondence $\Psi: \overline{\mathscr{B}} \rightarrow \overline{\mathscr{B}}$. Clearly this correspondence has a closed graph and nonempty convex values. Therefore, we are in the domain of application of the Kakutani-Fan-Glicksberg theorem (see [1, Chapter 16]). The correspondence has at least one fixed point, i.e. there exists an $r \in \overline{\mathscr{B}}$ such that $r \in \Psi(r)$. Now using the shape of the equations in (5.7) and the strong connectivity of the graph of successors $(\Sigma, \rightarrow)$, we obtain an $r \in \mathscr{B}$ (see [22, Theorem 4.5] for details). Let $\rho=\lambda A(r) /(\mu+\lambda C(r))$. The pair $(\rho, r)$ is an admissible solution to the TTE.

\subsection{The main results}

Lemma 5.4 begins to establish the link between the twisted traffic equations and the queue $\mathrm{M} / \mathrm{M} /(X, \Sigma)$.

Lemma 5.4. Let $(\rho, r)$ be an admissible solution to the TTE. Consider the zero-automatic queue of type $(X, \Sigma, \nu, r, \lambda, \mu)$. Let $Q_{r}$ be the infinitesimal generator of the queue-content process. Consider the measure $p_{\rho, r}$ on $L(X, \Sigma)$ defined by

$$
p_{\rho, r}\left(a_{n} \cdots a_{1}\right)=\rho^{n} \frac{r\left(a_{n}\right)}{r\left(\operatorname{Next}\left(a_{n}\right)\right)} \cdots \frac{r\left(a_{2}\right)}{r\left(\operatorname{Next}\left(a_{2}\right)\right)} r\left(a_{1}\right) \quad \text { for all } a_{n} \cdots a_{1} \in L(X, \Sigma) \text {. }
$$

We have $p_{\rho, r} Q_{r}=0$. Conversely, assume there exist a $\rho \in \mathbb{R}_{+}^{*}$ and an $r \in \mathcal{B}$ such that the measure $p_{\rho, r}$ defined by (5.8) satisfies $p_{\rho, r} Q_{r}=0$. Then $(\rho, r)$ is an admissible solution to the TTE.

Proof. We have $p_{\rho, r} Q_{r}=0$ if and only if, for all $u \in L(X, \Sigma)$,

$$
\sum_{\substack{v \in L(X, \Sigma) \\ v \neq u}} p_{\rho, r}(u) Q_{r}(u, v)=\sum_{\substack{v \in L(X, \Sigma) \\ v \neq u}} p_{\rho, r}(v) Q_{r}(v, u) .
$$


Denote the left- and right-hand side of the above equality by $L$ and $R$, respectively. Define

$$
\operatorname{Noact}(a)=\{b \in \Sigma \mid b * a=a\} .
$$

The left-hand side of (5.9) is then given by

$$
L= \begin{cases}\sum_{a \in \Sigma} \lambda \nu(a) r(\operatorname{Next}(a))=\lambda A(r) & \text { if } u=1_{\Sigma^{*}}, \\ p_{\rho, r}(u)\left(\lambda\left(1-v\left(\operatorname{Noact}\left(a_{n}\right)\right)\right)+\mu\right) & \text { otherwise. }\end{cases}
$$

For $u=1_{\Sigma^{*}}$, the right-hand side of (5.9) is given by

$$
R=\sum_{a \in \Sigma} \rho r(a)\left[\mu+\lambda \sum_{b * a=1_{X}} v(b)\right]=\rho(\mu+\lambda C(r)),
$$

and, for $u=a_{n} \cdots a_{1}, n \geq 1$,

$$
\begin{aligned}
& R= \lambda \nu\left(a_{n}\right) p_{\rho, r}\left(a_{n-1} \cdots a_{1}\right)+\sum_{\substack{d \neq a_{n} \\
b * d=a_{n}}} \lambda \nu(b) p_{\rho, r}\left(d a_{n-1} \cdots a_{1}\right) \\
&+\sum_{\substack{b \in \operatorname{Next}\left(a_{n}\right) \\
a * b=1_{X}}} \lambda \nu(a) p_{\rho, r}\left(b a_{n} \cdots a_{1}\right)+\sum_{b \in \operatorname{Next}\left(a_{1}\right)} p_{\rho, r}\left(a_{n} \cdots a_{1} b\right) \mu \\
&=p_{\rho, r}\left(a_{n} \cdots a_{1}\right)\left[\frac{1}{\rho} \frac{r\left(\operatorname{Next}\left(a_{n}\right)\right)}{r\left(a_{n}\right)} \lambda \nu\left(a_{n}\right)+\sum_{\substack{d \neq a_{n} \\
b * d=a_{n}}} \frac{r(d)}{r(\operatorname{Next}(d))} \frac{r\left(\operatorname{Next}\left(a_{n}\right)\right)}{r\left(a_{n}\right)} \lambda \nu(b)\right. \\
&\left.+\sum_{\substack{b \in \operatorname{Next}\left(a_{n}\right) \\
a * b=1_{X}}} \rho \lambda v(a) \frac{r(b)}{r(\operatorname{Next}(b))}+\sum_{b \in \operatorname{Next}\left(a_{1}\right)} \rho \frac{r(b)}{r\left(\operatorname{Next}\left(a_{1}\right)\right)} \mu\right] .
\end{aligned}
$$

Now recall that $b * d \in \Sigma$ implies that $\operatorname{Next}(b * d)=\operatorname{Next}(d)$. Thus, we obtain

$$
\begin{aligned}
R=p_{\rho, r}\left(a_{n} \cdots a_{1}\right)[ & \frac{1}{\rho} \frac{r\left(\operatorname{Next}\left(a_{n}\right)\right)}{r\left(a_{n}\right)} \lambda \nu\left(a_{n}\right)+\sum_{b * d=a_{n}} \frac{r(d)}{r\left(a_{n}\right)} \lambda \nu(b)-\lambda \nu\left(\operatorname{Noact}\left(a_{n}\right)\right) \\
& \left.+\sum_{\substack{b \in \operatorname{Next}\left(a_{n}\right) \\
a * b=1_{X}}} \rho \lambda \nu(a) \frac{r(b)}{r(\operatorname{Next}(b))}+\rho \mu\right]
\end{aligned}
$$

We see that for $u \neq 1_{\Sigma^{*}}$, the equality $L=R$ is precisely equivalent to the fact that $(\rho, r)$ is a solution to the TTE. For $u=1_{\Sigma^{*}}$, the equality $L=R$ is precisely equivalent to the fact that $\rho$ and $r$ satisfy (5.6). Therefore, the equality $L=R$ is precisely equivalent to the fact that $(\rho, r)$ is an admissible solution to the TTE. This completes the proof.

We now have all the ingredients to prove the central results of the paper. 
Theorem 5.1. Let $(X, \Sigma, \nu)$ be a plain triple. Fix $\lambda$ and $\mu$ in $\mathbb{R}_{+}^{*}$. Let $(\rho, r)$ be an admissible solution to the TTE. Consider the zero-automatic queue $(X, \Sigma, \nu, r, \lambda, \mu)$. Denote by $M_{r}=$ $\left(M_{r}(t)\right)_{t}$ the queue-content process and by $Q_{r}$ its infinitesimal generator. We have

$$
\begin{aligned}
& {[\rho<1] \Longleftrightarrow[\lambda \hat{\gamma}<\mu] \Longleftrightarrow M_{r} \text { is ergodic, }} \\
& {[\rho=1] \Longleftrightarrow[\lambda \hat{\gamma}=\mu] \Longleftrightarrow M_{r} \text { is null recurrent },} \\
& {[\rho>1] \Longleftrightarrow[\lambda \hat{\gamma}>\mu] \Longleftrightarrow M_{r} \text { is transient. }}
\end{aligned}
$$

Assume that $\lambda \hat{\gamma}<\mu$. The stationary distribution $\pi_{\rho, r}$ of the process $M_{r}$ is given by

$$
\pi_{\rho, r}\left(a_{n} \cdots a_{1}\right)=(1-\rho) p_{\rho, r}\left(a_{n} \cdots a_{1}\right)=(1-\rho) \rho^{n} q\left(a_{n}\right) \cdots q\left(a_{2}\right) r\left(a_{1}\right),
$$

for all $a_{n} \cdots a_{1} \in L(X, \Sigma)$, where $q(a)=r(a) / r(\operatorname{Next}(a))$ for all $a \in \Sigma$.

Proof. Let $(\rho, r)$ be an admissible solution to the TTE. Let $p_{\rho, r}$ be the measure defined in (5.8). Using the fact that $\sum_{a \in \Sigma} r(a)=1$, we have

$$
\sum_{u \in L(X, \Sigma)} p_{\rho, r}(u)=\sum_{n \in \mathbb{N}} \sum_{\substack{u \in L(X, \Sigma) \\|u|_{\Sigma}=n}} p_{\rho, r}(u)=\sum_{n \in \mathbb{N}} \rho^{n} .
$$

Hence, $\sum_{u} p_{\rho, r}(u)<\infty$ if and only if $\rho<1$. Now recall that $p_{\rho, r} Q_{r}=0$ from Lemma 5.4. It is standard (see, for instance, [4, Chapter 8]) that the process $M_{r}$ is ergodic if and only if $\sum_{u} p_{\rho, r}(u)<\infty$. Now, according to Proposition 4.1, $M_{r}$ is ergodic if and only if $\lambda \hat{\gamma}<\mu$. By combining the three equivalences, we obtain

$$
[\rho<1] \Longleftrightarrow\left[M_{r} \text { is ergodic }\right] \Longleftrightarrow[\lambda \hat{\gamma}<\mu] .
$$

The result in (5.10) holds as a direct consequence of Lemma 5.4.

Now let us turn our attention to the null recurrent case. Assume that $\rho=1$. Using Lemma 5.2 and Proposition 4.1, we have

$$
[\rho=1] \Longrightarrow[\lambda \hat{\gamma}=\mu] \Longleftrightarrow M_{r} \text { is null recurrent. }
$$

Let $(\tilde{\rho}, \tilde{r})$ be an admissible solution to the TTE. Using the argumentation in the forthcoming proof of Theorem 5.2, we deduce that we must have $\tilde{\rho}=\rho=1$. Hence, we have an equivalence on the left of (5.11). This completes the proof.

Assume that $\lambda \hat{\gamma}=\mu$. It follows immediately from Lemma 5.2 and Theorem 5.1 that $(1, \hat{r})$ is the unique admissible solution to the TTE. We now prove a more interesting result in the same vein.

Theorem 5.2. Consider the same model as in Theorem 5.1. Assume that $\lambda \hat{\gamma}<\mu$. Then the TTE have a unique admissible solution. In particular, there is only one variant of the zero-automatic queuе $\mathrm{M} / \mathrm{M} /(X, \Sigma)$ with a product form distribution.

Proof. Let $(\rho, r)$ and $(\tilde{\rho}, \tilde{r})$ be two admissible solutions to the TTE. According to Theorem 5.1, we have $\rho<1$ and $\tilde{\rho}<1$. Let $\pi$ and $\tilde{\pi}$ be the respective stationary distributions of $M_{r}$ and $M_{\tilde{r}}$. We now use the following classical result of ergodic Markov processes (see, for instance, $[4$, Chapter 8 , Theorem 5.1]): the stationary distribution is proportional to the time spent in each state in an excursion of the process from $s$ to $s$, for some arbitrary state $s$. Assume 
that the queue-content is $1_{\Sigma^{*}}$ at instant 0 . Let $T$ and $\tilde{T}$ be the first instant of jump of $M_{r}$ and $M_{\tilde{r}}$, respectively. Let $R$ and $\tilde{R}$ be the first return instant to $1_{\Sigma^{*}}$ of $M_{r}$ and $M_{\tilde{r}}$, respectively. For all $u \in L(X, \Sigma)$, we have

$$
\pi(u) \sim \mathrm{E}\left[\int_{0}^{R} \mathbf{1}_{\left\{M_{r}(t)=u\right\}} \mathrm{d} t\right], \quad \tilde{\pi}(u) \sim \mathrm{E}\left[\int_{0}^{\tilde{R}} \mathbf{1}_{\left\{M_{\tilde{r}}(t)=u\right\}} \mathrm{d} t\right],
$$

where ' $\sim$ ' stands for 'proportional to'. For all $u \in L(X, \Sigma)$ and $u \neq 1_{\Sigma^{*}}$, it follows that

$$
\pi(u) \sim \mathrm{E}\left[\int_{T}^{R} \mathbf{1}_{\left\{M_{r}(t)=u\right\}} \mathrm{d} t\right], \quad \tilde{\pi}(u) \sim \mathrm{E}\left[\int_{\tilde{T}}^{\tilde{R}} \mathbf{1}_{\left\{M_{\tilde{r}}(t)=u\right\}} \mathrm{d} t\right] .
$$

And, conditioning by the values of $M_{r}(T)$ and $M_{\tilde{r}}(\tilde{T})$, respectively, we have, for all $u \in L(X, \Sigma)$ and $u \neq 1_{\Sigma^{*}}$,

$$
\begin{aligned}
& \pi(u) \sim \sum_{a \in \Sigma} v(a) r(\operatorname{Next}(a)) \mathrm{E}\left[\mathbf{1}_{\left\{M_{r}(T)=a\right\}} \int_{T}^{R} \mathbf{1}_{\left\{M_{r}(t)=u\right\}} \mathrm{d} t\right], \\
& \tilde{\pi}(u) \sim \sum_{a \in \Sigma} v(a) \tilde{r}(\operatorname{Next}(a)) \mathrm{E}\left[\mathbf{1}_{\left\{M_{\tilde{r}}(\tilde{T})=a\right\}} \int_{\tilde{T}}^{\tilde{R}} \mathbf{1}_{\left\{M_{\tilde{r}}(t)=u\right\}} \mathrm{d} t\right] .
\end{aligned}
$$

Observe that the generators $Q_{r}$ and $Q_{\tilde{r}}$ differ only in the line indexed by $1_{\Sigma^{*}}$. In other words, the conditional law of $\left(M_{r}(t)\right)_{t \in[T, R]}$ on the event $\left\{M_{r}(T)=a\right\}$ is equal to the conditional law of $\left(M_{\tilde{r}}(t)\right)_{t \in[\tilde{T}, \tilde{R}]}$ on the event $\left\{M_{\tilde{r}}(\tilde{T})=a\right\}$. Therefore, $\pi$ and $\tilde{\pi}$ are obtained as linear combinations of the same measures $p_{a}$ defined by

$$
p_{a}(u)=\mathrm{E}\left[\mathbf{1}_{\left\{M_{r}(T)=a\right\}} \int_{T}^{R} \mathbf{1}_{\left\{M_{r}(t)=u\right\}} \mathrm{d} t\right]=\mathrm{E}\left[\mathbf{1}_{\left\{M_{\tilde{r}}(\tilde{T})=a\right\}} \int_{\tilde{T}}^{\tilde{R}} \mathbf{1}_{\left\{M_{\tilde{r}}(t)=u\right\}} \mathrm{d} t\right] .
$$

For $n \in \mathbb{N}^{*}$, set $\pi(n)=\pi\left\{\left.u \in L(X, \Sigma)|| u\right|_{\Sigma}=n\right\}$. Define $\tilde{\pi}(n)$ and $p_{a}(n)$ accordingly. Using the above, for all $n \in \mathbb{N}^{*}$, we have

$$
\pi(n) \sim \sum_{a \in \Sigma} v(a) r(\operatorname{Next}(a)) p_{a}(n), \quad \tilde{\pi}(n) \sim \sum_{a \in \Sigma} v(a) \tilde{r}(\operatorname{Next}(a)) p_{a}(n) .
$$

Besides, according to (5.10) we have $\pi(n) \sim \rho^{n}$ and $\tilde{\pi}(n) \sim \tilde{\rho}^{n}$. In view of (5.12), we easily conclude that we must have $\rho=\tilde{\rho}$. It remains to prove that $r=\tilde{r}$. Let us first show that

$$
\text { [for all } a \in \Sigma, q(a)=\tilde{q}(a)] \Longrightarrow[\text { for all } a \in \Sigma, r(a)=\tilde{r}(a)] \text {. }
$$

We have $q(a)=r(a) / r(\operatorname{Next}(a))$, for all $a$. We can reinterpret this as $M r=r$, with $r$ being viewed as a column vector and with $M$ being the matrix of dimension $\Sigma \times \Sigma$ defined by

$$
M_{a, b}= \begin{cases}q(a) & \text { if } b \in \operatorname{Next}(a) \\ 0 & \text { otherwise }\end{cases}
$$

for all $a, b \in \Sigma$. Consequently, the matrix $M$ is irreducible. As $r$ has all positive entries, the Perron-Frobenius theorem asserts that $r$ is necessarily the Perron eigenvector of the matrix $M$, i.e. the unique (up to a multiplicative constant) eigenvector associated with the spectral radius. 
However, we also have $M \tilde{r}=\tilde{r}$ and $\sum_{a} r(a)=\sum_{a} \tilde{r}(a)=1$. By uniqueness of the Perron eigenvector, we conclude that $r=\tilde{r}$.

It remains to prove that $q(a)=\tilde{q}(a)$ for all $a \in \Sigma$. Define $C(X, \Sigma) \subset L(X, \Sigma)$ by

$$
C(X, \Sigma)=\left\{u_{1} \cdots u_{k} \in L(X, \Sigma) \mid u_{1} \in \operatorname{Next}\left(u_{k}\right)\right\}
$$

Observe that $[u \in C(X, \Sigma)]$ implies that $\left[u^{n} \in C(X, \Sigma)\right.$ for all $\left.n\right]$. For $u=u_{1} \cdots u_{k} \in$ $C(X, \Sigma)$, let $q(u)=q\left(u_{1}\right) \cdots q\left(u_{k}\right)$. Define $\tilde{q}(u)$ analogously. Using (5.10), for all $n \in \mathbb{N}^{*}$ and for some constants $C_{1}, C_{2}$, we have

$$
\pi\left(u^{n}\right)=C_{1} \rho^{n|u|_{\Sigma}} q(u)^{n} r\left(\operatorname{Next}\left(u_{k}\right)\right), \quad \tilde{\pi}\left(u^{n}\right)=C_{2} \rho^{n|u| \Sigma} \tilde{q}(u)^{n} \tilde{r}\left(\operatorname{Next}\left(u_{k}\right)\right) .
$$

Moreover,

$$
\pi\left(u^{n}\right)=C_{3} \sum_{a \in \Sigma} v(a) r(\operatorname{Next}(a)) p_{a}\left(u^{n}\right) \quad \text { and } \quad \tilde{\pi}\left(u^{n}\right)=C_{4} \sum_{a \in \Sigma} v(a) \tilde{r}(\operatorname{Next}(a)) p_{a}\left(u^{n}\right),
$$

for some constants $C_{3}, C_{4}$. Therefore, $\pi\left(u^{n}\right)$ and $\tilde{\pi}\left(u^{n}\right)$ must grow at the same exponential rate as a function of $n$. In view of (5.13), we must have

$$
q\left(u_{1}\right) \cdots q\left(u_{k}\right)=\tilde{q}\left(u_{1}\right) \cdots \tilde{q}\left(u_{k}\right) .
$$

For the remaining step, the argument depends on the form of $X$. Let $X=X_{1} \star \cdots \star X_{K} \star X_{K+1} \star F$, with $X_{K+1}=\Sigma_{K+1}^{*}\left(\left|\Sigma_{K+1}\right| \geq 0\right), F=F\left(\Sigma_{K+2}\right)\left(\left|\Sigma_{K+2}\right| \geq 0\right), X_{i}$ being finite monoids for $1 \leq i \leq K$, and $\Sigma=\Sigma_{K+2}^{-1} \sqcup_{1 \leq i \leq K+2} \Sigma_{i}$, where $\Sigma_{i}=\left(X_{i} \backslash\left\{1_{X_{i}}\right\}\right)$ for $1 \leq i \leq K$. For $a \in \Sigma_{i}$ and $1 \leq i \leq K, \operatorname{Next}(a)=\Sigma \backslash \Sigma_{i}$. For $b \in \Sigma_{K+1}, \operatorname{Next}(b)=\Sigma$. For $c \in \Sigma_{K+2}$, $\operatorname{Next}(c)=\Sigma \backslash\left\{c^{-1}\right\}$.

We first treat the case in which $\left|\Sigma_{K+1}\right|+\left|\Sigma_{K+2}\right| \geq 1$. Then there exists an $i \in\{K+1, K+2\}$ such that $\left|\Sigma_{i}\right| \geq 1$. Set Gen $=\Sigma_{K+1} \sqcup \Sigma_{K+2} \sqcup \Sigma_{K+2}^{-1}$. For all $a \in \operatorname{Gen}, a \in \operatorname{Next}(a)$; thus, we have $q(a)^{2}=\tilde{q}(a)^{2}$. This implies that $q(a)=\tilde{q}(a)$. Consider $b \in \Sigma \backslash$ Gen and $a \in$ Gen, we have $b \in \operatorname{Next}(a)$, so, according to (5.14), $q(b) q(a)=\tilde{q}(b) \tilde{q}(a)$. Hence, $q(b)=\tilde{q}(b)$. So $q(c)=\tilde{q}(c)$ for all $c \in \Sigma$.

We now consider the case in which $\left|\Sigma_{K+1}\right|+\left|\Sigma_{K+2}\right|=0$. Assume that $K \geq 3$. Consider $a \in \Sigma_{1}, b \in \Sigma_{2}$, and $c \in \Sigma_{3}$. We have $\operatorname{Next}(a)=\Sigma \backslash \Sigma_{1}, \operatorname{Next}(b)=\Sigma \backslash \Sigma_{2}$, and $\operatorname{Next}(c)=\Sigma \backslash \Sigma_{3}$. Therefore, $a b c \in C(X, \Sigma)$ and $b c \in C(X, \Sigma)$. Using (5.14), we deduce that $q(a) q(b) q(c)=\tilde{q}(a) \tilde{q}(b) \tilde{q}(c)$ and $q(b) q(c)=\tilde{q}(b) \tilde{q}(c)$. Thus, we conclude that $q(a)=\tilde{q}(a)$ for all $a \in \Sigma$.

Assume now that $K=2$. The above argument no longer holds. First of all, we want to prove that

$$
\frac{r(a)}{r\left(\Sigma_{i}\right)}=\frac{\tilde{r}(a)}{\tilde{r}\left(\Sigma_{i}\right)} \quad \text { for all } a \in \Sigma_{i}
$$

Consider $a, b \in \Sigma_{1}$ and $c \in \Sigma_{2}$. We have $\operatorname{Next}(a)=\operatorname{Next}(b)=\Sigma_{2}$ and $\operatorname{Next}(c)=\Sigma_{1}$. Hence, $a c \in C(X, \Sigma)$ and $b c \in C(X, \Sigma)$. Using (5.14), we have $q(a) q(c)=\tilde{q}(a) \tilde{q}(c)$ and $q(b) q(c)=\tilde{q}(b) \tilde{q}(c)$. This implies that $q(a) / q(b)=\tilde{q}(a) / \tilde{q}(b)$, which is equivalent to (5.15). Let

$$
R(a)=\frac{r(a)}{r\left(\Sigma_{i}\right)}=\frac{\tilde{r}(a)}{\tilde{r}\left(\Sigma_{i}\right)} \quad \text { if } a \in \Sigma_{i}
$$


Now we sum the TTE corresponding to all the elements of $\Sigma_{1}$ and perform the simplifications implied by (5.15). For instance, for $(\rho, r)$, we obtain

$$
\begin{aligned}
\rho(\lambda+\mu) r\left(\Sigma_{1}\right)= & \rho^{2} \mu r\left(\Sigma_{1}\right)+\lambda \nu\left(\Sigma_{1}\right) r\left(\Sigma_{2}\right)+\rho \lambda \sum_{a * b \in \Sigma_{1}} v(a) r(b) \\
& +\rho^{2} \lambda \sum_{a \in \Sigma_{1}} \sum_{\substack{b, d \in \Sigma_{2} \\
b * d=1_{X_{2}}}} v(b) \frac{r(d)}{r\left(\Sigma_{1}\right)} r(a) \\
= & \rho^{2} \mu r\left(\Sigma_{1}\right)+\lambda \nu\left(\Sigma_{1}\right) r\left(\Sigma_{2}\right)+\rho \lambda r\left(\Sigma_{1}\right) \sum_{a * b \in \Sigma_{1}} v(a) R(b) \\
& +\rho^{2} \lambda r\left(\Sigma_{2}\right) \sum_{\substack{b, d \in \Sigma_{2} \\
b * d=1_{X_{2}}}} v(b) R(d) .
\end{aligned}
$$

Let $B_{1}=\sum_{a * b \in \Sigma_{1}} v(a) R(b)$ and $C_{2}=\sum_{a, b \in \Sigma_{2}, a * b=1_{X_{2}}} v(a) R(b)$. Using the fact that $r\left(\Sigma_{2}\right)=1-r\left(\Sigma_{1}\right)$, we obtain

$$
r\left(\Sigma_{1}\right)=\frac{\lambda \nu\left(\Sigma_{1}\right)+\rho^{2} \lambda C_{2}}{\rho(\lambda+\mu)-\rho^{2} \mu+\lambda \nu\left(\Sigma_{1}\right)-\rho \lambda B_{1}+\rho^{2} \lambda C_{2}} .
$$

All the terms on the right-hand side of (5.16) are unchanged when we write the corresponding equation for $\tilde{r}$. Thus, $r\left(\Sigma_{1}\right)=\tilde{r}\left(\Sigma_{1}\right)$. In view of (5.15), we deduce that $r(a)=\tilde{r}(a)$ for all $a \in \Sigma$. This completes the proof.

When the triple is not plain, the TTE may have several admissible solutions. This is, for instance, the case for the triple $\left(F(a),\left\{a, a^{-1}\right\},\left\{\frac{1}{2}, \frac{1}{2}\right\}\right)$ as discussed in Section 6.2.4.

Remark 5.1. In the case $\rho<1$, if the boundary condition is chosen according to $r^{\prime}$, where $\left(\rho, r^{\prime}\right)$ is not a solution to the TTE, then the stationary distribution of $M_{r^{\prime}}$ exists (Proposition 4.1). However, we do not know how to compute it exactly. See Remark 3.1 for a justification of the form of the boundary condition.

5.2.1. Poisson departure processes. The celebrated Burke's theorem states that the departure process from a stable $\mathrm{M} / \mathrm{M} / 1$ queue is a Poisson process of the same rate as the arrival process. A nice consequence of Theorem 5.1 is that an analog of Burke's theorem holds for zero-automatic queues.

In a zero-automatic queue, 'departures' occur both at the front-end and at the back-end of the buffer. Here we consider only the front-end departures, i.e. the ones corresponding to service completions and not to buffer cancellations. Let $M=(M(t))_{t}$ be the queue-content process of some zero-automatic queue $\mathrm{M} / \mathrm{M} /(X, \Sigma)$. A departure is an instant of jump of $M$ corresponding to a jump of the type $u_{n} \cdots u_{1} \rightarrow u_{n} \cdots u_{2}$ for $u=u_{n} \cdots u_{1} \in L(X, \Sigma) \backslash\left\{1_{\Sigma^{*}}\right\}$. When $u=a^{n}, a \in \Sigma$ and $n \geq 1$ (the case (3.1)-(3.3) in Definition 3.1), special care must be taken to define departures. The jumps of type $a^{n} \rightarrow a^{n-1}$ which are departures occur at a rate $\mu$. The departure process is the point process of departures.

Theorem 5.3. The model is the same as in Theorem 5.1. Assume that $\lambda \hat{\gamma}<\mu$. Let $(\rho, r)$ be an admissible solution of the TTE. Consider the zero-automatic queue $(X, \Sigma, \nu, r, \lambda, \mu)$. The stationary departure process is a Poisson process of rate $\rho \mu$. Furthermore, for all $t$, the queue-content at time $t$ is independent of the departure process up to time $t$. 
Proof. The simplest proof of Burke's theorem uses reversibility and is due to Reich; see, e.g. [17] for details. We use a similar argument here.

Let $M_{r}$ be the stationary queue-content process. Its marginal distribution at a given instant is $\pi_{\rho, r}$ given in (5.10). Let $D$ be the corresponding departure process. By definition, the instantaneous rate of $D$ is $c(t)=\mu$ if $M_{r}(t) \neq 1_{\Sigma^{*}}$ and $c(t)=0$ otherwise.

Now let us consider the time-reversed point process $\tilde{D}$. The process $\tilde{D}$ corresponds to the instants of 'right-increase' of the time-reversed process $\left(\tilde{M}_{r}(t)\right)_{t}$. Therefore, the instantaneous rate $\tilde{c}(t)$ of $\tilde{D}$ is as follows. If $\tilde{M}_{r}(t)=a_{n} \cdots a_{1} \in L(X, \Sigma) \backslash\left\{1_{\Sigma^{*}}\right\}$, we have

$$
\tilde{c}(t)=\sum_{a \in \operatorname{Next}\left(a_{1}\right)} \frac{\pi_{\rho, r}\left(a_{n} \cdots a_{1} a\right)}{\pi_{\rho, r}\left(a_{n} \cdots a_{1}\right)} \mu=\sum_{a \in \operatorname{Next}\left(a_{1}\right)} \frac{\rho r(a)}{r\left(\operatorname{Next}\left(a_{1}\right)\right)} \mu=\rho \mu,
$$

and if $\tilde{M}_{r}(t)=1_{\Sigma^{*}}$, we have

$$
\tilde{c}(t)=\sum_{a \in \Sigma} \frac{\pi_{\rho, r}(a)}{\pi_{\rho, r}\left(1_{\Sigma^{*}}\right)} \mu=\sum_{a \in \Sigma} \rho r(a) \mu=\rho \mu .
$$

We conclude that $\tilde{D}$ is a Poisson process of rate $\rho \mu$. As Poisson processes are preserved by time-reversal, $D$ is also a Poisson process of rate $\rho \mu$. Also, using the Markov property of Poisson processes, $\tilde{M}_{r}(t)$ is independent of the process $\tilde{D}$ after time $t$. Under time-reversal, this translates as $M_{r}(t)$ is independent of the departure process $D$ up to time $t$. This completes the proof.

Here are some additional comments on Theorem 5.3.

(1) The infinitesimal generator of the time-reversed process $\tilde{M}_{r}$ is certainly not the infinitesimal generator of a zero-automatic queue. This was already the case for the G-queue. However, this is in contrast with the situation for the $\mathrm{M} / \mathrm{M} / 1$ queue.

(2) For $a \in \Sigma$, the departure process $D_{a}$ of customers of class $a$ is not a Poisson process.

(3) Equation (5.17) corresponds to the condition defining 'quasi-reversibility' in Chao et al. [6, Definition 3.4].

(4) The saturation principle of Baccelli and Foss [3] holds for many classical queueing systems. We provide a brief outline of it here.

Consider a queueing system with an infinite capacity buffer. Let $\mu_{0}$ be the departure rate in the saturated system in which an infinite number of customers are stacked in the buffer. Now, if the actual arrival rate in the system is $\lambda<\mu_{0}$, then the system is stable, and the departure rate is $\lambda$. A dual presentation of the same principle is as follows. Let $\lambda_{0}$ be the growth rate of the buffer in the blocked system where the server has been shut down. If the actual service rate in the system is $\mu>\lambda_{0}$, then the system is stable, and the departure rate is $\lambda_{0}$.

Zero-automatic queues do not satisfy the saturation principle. This can be viewed using the following inequalities (to be deduced from Theorem 5.3):

$$
\lambda \hat{\gamma} \leq \rho \mu<\mu
$$

where $\rho \mu$ is the actual equilibrium departure rate $\mu$ is the departure rate from the saturated system, and $\lambda \hat{\gamma}$ is the growth rate of the buffer in the blocked system. 


\subsection{Quasi-birth-and-death processes}

Quasi-birth-and-death (QBD) processes appear naturally in the modelling of several queueing and communication systems. As such, they have been extensively studied; see, for instance, the monographs [20], [24]. The results in Section 5.2 can be put in perspective by considering the relation between zero-automatic queues and QBD processes. To this end, we define an 'approximated' and quite simplified version of the zero-automatic queue. The idea is to keep track of the queue-content only through the number of customers and the class of the back-end customer. Clearly a difficulty arises: if a cancellation occurs at the back-end of the buffer, there is no way to retrieve the class of the new back-end customer. This missing information is compensated as follows: the class is chosen at random according to the relevant conditional law.

Consider a zero-automatic queue. The notations and assumptions are the same as those in Theorem 5.1. In particular $(\rho, r)$ is an admissible solution to the TTE. We assume that $\lambda \hat{\gamma}<\mu$. Recall that $Q_{r}$ is the infinitesimal generator of the queue-content on the state space $L(X, \Sigma)$, and that $\pi_{\rho, r}$ given by (5.10) is its stationary distribution. Consider the following application:

$$
\begin{aligned}
f: L(X, \Sigma) & \longrightarrow\{0\} \cup\left(\mathbb{N}^{*} \times \Sigma\right), \\
u_{n} \cdots u_{1} & \longmapsto\left(n, u_{n}\right), \\
1_{\Sigma^{*}} & \longmapsto 0 .
\end{aligned}
$$

Define the infinitesimal generator $\tilde{Q}_{r}$ on the state space $\{0\} \cup\left(\mathbb{N}^{*} \times \Sigma\right)$ by

$$
\tilde{Q}_{r}(x, y)=\sum_{u \in f^{-1}(x)} \frac{\pi_{\rho, r}(u)}{\pi_{\rho, r}\left(f^{-1}(x)\right)} \sum_{v \in f^{-1}(y)} Q_{r}(u, v) .
$$

Using (5.10) and simplifying, we obtain

$$
\tilde{Q}_{r}((n, a) ;(n-1, b))=\lambda \sum_{c * a=1_{X}} v(c) \frac{r(b)}{r(\operatorname{Next}(a))} \mathbf{1}_{\{b \in \operatorname{Next}(a)\}} .
$$

Define a total order on $\{0\} \cup\left(\mathbb{N}^{*} \times \Sigma\right)$ as follows: 0 is the smallest element and $(n, a) \leq(m, b)$ if $n<m$ or $(n=m, a \preceq b)$, where ' $\preceq$ ' is some total order on $\Sigma$. A couple of lines of computation is required to check the following. If rows and columns are ranked according to the above order, then the infinitesimal generator $\tilde{Q}_{r}$ is block tridiagonal of the form

$$
\tilde{Q}_{r}=\left(\begin{array}{ccccc}
a & A(r) & 0 & 0 & \\
B & A_{1} & A_{0} & 0 & \ddots \\
0 & A_{2}(r) & A_{1} & A_{0} & \ddots \\
0 & 0 & A_{2}(r) & A_{1} & \ddots \\
& \ddots & \ddots & \ddots & \ddots
\end{array}\right),
$$

where $a$ is of dimension $1 \times 1, A(r)$ is of dimension $1 \times \Sigma, B$ is of dimension $\Sigma \times 1$, and $A_{0}, A_{1}$, and $A_{2}(r)$ are of dimension $\Sigma \times \Sigma$. Furthermore, the entries of $B, A_{0}$, and $A_{1}$ can be expressed as a function of $\lambda, \mu$, and $v$, and the entries of $A(r)$ and $A_{2}(r)$ can be expressed as a function of $\lambda, \mu, v$, and $r$. 
According to the terminology in Neuts [24], $\tilde{Q}_{r}$ is the infinitesimal generator of a QBD process with complex boundary behavior. For any such ergodic process, the shape of the stationary distribution is known; see, e.g. [24, Chapter 1.5]. Thus, we assume that $\tilde{Q}_{r}$ is ergodic and we apply the general results to obtain the stationary distribution $\tilde{\pi}$, given by

$$
\tilde{\pi}(0)=y, \quad \text { for all } n \geq 1, \text { for all } a \in \Sigma, \tilde{\pi}(n, a)=\left(x R^{n-1}\right)(a),
$$

where

$$
\begin{aligned}
& A_{0}+R A_{1}+R^{2} A_{2}(r)=0, \\
& a y+x B=0, \quad y A(r)+x\left(A_{1}+R A_{2}(r)\right)=0, \\
& y+x(I-R)^{-1}(1, \ldots, 1)^{T}=1 .
\end{aligned}
$$

In (5.19)-(5.21), $R$ is a matrix of dimension $\Sigma \times \Sigma$ and is the minimal nonnegative solution to the first equation. The pair $(y, x)$, where $y$ is a scalar and $x$ is a row vector of dimension $\Sigma$, is the unique positive solution to (5.20) and (5.21).

The stationary distribution $\tilde{\pi}$ given in $(5.18)$ has a matrix product form. This matrix product form is said to be a product form (this is called level-geometric with parameter 0 in [10]) if $\tilde{\pi}(n, a)=\eta^{n-1} \tilde{\pi}(1, a)=\eta^{n-1} x(a)$, for some $\eta \in(0,1)$. Clearly, a necessary and sufficient condition for this to hold is $x R=\eta x$. Assume that this last equality holds. By multiplying (5.19)-(5.21) by $x$, and by simplifying the equations, we obtain

$$
\begin{aligned}
& x\left(A_{0}+\eta A_{1}+\eta^{2} A_{2}(r)\right)=0, \\
& a y+x B=0, \quad y A(r)+x\left(A_{1}+\eta A_{2}(r)\right)=0, \\
& y+(1-\eta)^{-1} x(1, \ldots, 1)^{T}=1 .
\end{aligned}
$$

Now let us replace $x$ by $c r$, where $c \in \mathbb{R}$, and $\eta$ by $\rho$ in (5.22)-(5.24). Equation (5.22) yields precisely the TTE (5.1) for the pair $(\rho, r)$. The other equations in (5.23) and (5.24) yield exactly $y=1-\rho$ and $c=\rho(1-\rho)$. We conclude that the stationary distribution of $\tilde{Q}_{r}$ is given by

$$
\tilde{\pi}(0)=1-\rho, \quad \text { for all } n \geq 1, \text { for all } a \in \Sigma, \tilde{\pi}(n, a)=(1-\rho) \rho^{n} r(a) .
$$

This is coherent with the form of $\pi_{\rho, r}$ given in (5.10). Thus, the above provides an a posteriori and partial justification for the shape of $\pi_{\rho, r}$. It also provides further insight into the central role of the TTE.

Classically in QBD theory [20], [10], the boundary condition is modified to obtain a product form stationary distribution. Here, the situation is more complex as both the boundary condition and the $A_{2}$ matrix are modified in the quest for the product form. Observe also that the result of Theorem 5.1 is much deeper than (5.25). In particular, there is a priori no way to retrieve the result on $Q_{r}$ from the one on the simplified generator $\tilde{Q}_{r}$.

\section{Extension and examples}

\subsection{Zero-automatic queues built on zero-automatic pairs}

All the results in Sections 4 and 5 are derived for queues built on plain monoids not isomorphic to $\mathbb{Z}$ (Definition 2.1). However, the zero-automatic queue built on $\mathbb{Z}$ is interesting in itself (it corresponds to Gelenbe's G-queue, see Section 3) and exhibits new phenomena. It is therefore worthwhile to determine a subset of the above results which remain true for this queue. In 
fact, we define a more general framework and introduce the notion of zero-automatic triples, extending the plain triples of Definition 2.1.

Let $(X, *)$ be a group or monoid with a set of generators $\Sigma$. Denote by $\pi: \Sigma^{*} \rightarrow X$ the monoid homomorphism which associates to a word $a_{1} \cdots a_{k}$ of $\Sigma^{*}$ the element $a_{1} * \cdots * a_{k}$ of $X$. A language $L$ of $\Sigma^{*}$ is a cross-section of $X$ if the restriction of $\pi$ to $L$ is a bijection. The inverse map $\Phi: X \rightarrow L$ is then called the normal form map. Define the language $L(X, \Sigma) \subset \Sigma^{*}$ as in (2.2). Define the sets

$$
\operatorname{Left}(a)=\left\{b \in \Sigma \mid b * a \notin \Sigma \cup\left\{1_{X}\right\}\right\}, \quad \operatorname{Right}(a)=\left\{b \in \Sigma \mid a * b \notin \Sigma \cup\left\{1_{X}\right\}\right\},
$$

for all $a \in \Sigma$. In the case of a plain monoid with natural generators, we have Left $=$ Right $=$ Next; see (2.3).

Definition 6.1. Let $(G, *)$ be a group with a finite set of generators $\Sigma$. We say that the pair $(G, \Sigma)$ is zero-automatic if $L(G, \Sigma)$ is a cross-section of $\mathrm{G}$.

Such pairs were first considered by Stallings [27] under another name. It can be proved, using the results from Stallings, that $G$ is necessarily isomorphic to a plain group. However, the set $\Sigma$ may be larger than a natural (see Section 2.2) set of generators of the plain group. Now let us extend the notion to monoids. To obtain good properties it is necessary to choose a more complex definition, first proposed in [22] and recalled below.

Definition 6.2. Let $(M, *)$ be a monoid with a finite set of generators $\Sigma$. Assume that $L(M, \Sigma)$ is a cross-section. Let $\Phi: M \rightarrow L(M, \Sigma)$ be the corresponding normal form map. Assume, for all $u \in M$ such that $\Phi(u)=u_{1} \cdots u_{k}$ and for all $a \in \Sigma$, that

$$
\begin{gathered}
\Phi(u * a)= \begin{cases}u_{1} \cdots u_{k-1} & \text { if } u_{k} * a=1_{M}, \\
u_{1} \cdots u_{k-1} v & \text { if } u_{k} * a=v \in \Sigma, \\
u_{1} \cdots u_{k} a & \text { otherwise, }\end{cases} \\
\Phi(a * u)= \begin{cases}u_{2} \cdots u_{k} & \text { if } a * u_{1}=1_{M}, \\
v u_{2} \cdots u_{k} & \text { if } a * u_{1}=v \in \Sigma, \\
a u_{1} \cdots u_{k} & \text { otherwise. }\end{cases}
\end{gathered}
$$

Furthermore, for all $a, b \in \Sigma$ such that $a * b \in \Sigma$, assume that

$$
\operatorname{Left}(a * b)=\operatorname{Left}(a), \quad \operatorname{Right}(a * b)=\operatorname{Right}(b) .
$$

Then we say that the pair $(M, \Sigma)$ is zero-automatic.

In the group case $M=G,(6.1)$ and (6.2) are implied by the fact that the language $L(G, \Sigma)$ is a cross-section.

The pairs formed by a plain monoid and natural generators are zero-automatic. However, in contrast with the group case, plain monoids do not exhaust the family of monoids appearing in zero-automatic pairs. For instance, $(M=\langle a, b \mid a b=1\rangle,\{a, b\})$ is a zero-automatic pair, but the monoid $M$ is not isomorphic to a plain monoid. This example is studied further in Section 6.2.

Definition 6.3. A triple $(X, \Sigma, v)$ is said to be zero-automatic if

(i) $(X, \Sigma)$ is a zero-automatic pair with $X$ infinite;

(ii) $v$ is a probability measure whose support is included in $\Sigma$ and generates $X$. 
Any plain triple, see Definition 2.1, is zero-automatic. Observe that $(X, v)$ may not be transient in a zero-automatic triple. Also, the graph of succesors $(\Sigma, \rightarrow)$ defined in (2.4) may not be strongly connected.

Consider a zero-automatic triple $(X, \Sigma, v), \lambda, \mu \in \mathbb{R}_{+}^{*}$, and $r \in \overline{\mathscr{B}}$ (see (2.6)). The zeroautomatic queue of type $(X, \Sigma, v, r, \lambda, \mu)$ is defined exactly as in Definition 3.1. However, the definition of the TE is modified as follows:

$$
x(a)=v(a) x(\operatorname{Right}(a))+\sum_{b * d=a} v(b) x(d)+\sum_{\substack{d \in \operatorname{Left}(a) \\ b * d=1_{X}}} v(b) \frac{x(d)}{x(\operatorname{Right}(d))} x(a) .
$$

The definition of the TTE is also modified as follows:

$$
\begin{aligned}
\eta(\lambda+\mu) x(a)= & \eta^{2} \mu x(a)+\lambda v(a) x(\operatorname{Right}(a))+\eta \lambda \sum_{b * d=a} v(b) x(d) \\
& +\eta^{2} \lambda \sum_{\substack{d \in \operatorname{Left}(a) \\
b * d=1_{X}}} v(b) \frac{x(d)}{x(\operatorname{Right}(d))} x(a) .
\end{aligned}
$$

We use the convention described after (2.6) to define a solution to the TTE belonging to $\overline{\mathcal{B}}$. The TE do not necessarily have a unique solution, in contrast with Proposition 2.2. The TTE also do not necessarily have a unique solution and a solution $r \in \overline{\mathcal{B}}$ to the TTE does not necessarily belong to $\mathscr{B}$. This is in contrast with Theorem 5.2. An analog of Lemma 5.1 holds: if $(\rho, r)$, $r \in \bar{B}$, is a solution to the TTE then either $(\rho, r)=(1, r)$ and $r$ is a solution to the TE, or

$$
\rho=\frac{\lambda A(r)}{\mu+\lambda C(r)} \text {. }
$$

We can now state the following result. Observe in particular that there may be several variants (corresponding to different $r \mathrm{~s}$ ) of the zero-automatic queue with a product form.

Proposition 6.1. Let $(X, \Sigma, v)$ be a zero-automatic triple. Let $(\rho, r), r \in \bar{B}$, be a solution to the TTE satisfying (6.3). Assume that $r(a)>0$ implies that $r(\operatorname{Right}(a))>0$, for all $a \in \Sigma$. Define $\tilde{\Sigma}=\{a \in \Sigma \mid r(a)>0\}$ and $\tilde{L}=L(X, \Sigma) \cap \tilde{\Sigma}^{*}$. Consider the zero-automatic queue of type $(X, \Sigma, v, r, \lambda, \mu)$. Let $Q_{r}$ be the infinitesimal generator of the queue-content process $M_{r}$. Consider the measure $p_{\rho, r}$ on $\tilde{L}$ defined by

$$
p_{\rho, r}\left(a_{n} \cdots a_{1}\right)=\rho^{n} \frac{r\left(a_{n}\right)}{r\left(\operatorname{Right}\left(a_{n}\right)\right)} \cdots \frac{r\left(a_{2}\right)}{r\left(\operatorname{Right}\left(a_{2}\right)\right)} r\left(a_{1}\right),
$$

for all $a_{n} \cdots a_{1} \in \tilde{L}$. We have $p_{\rho, r} Q_{r}=0$. Besides, we have

$$
\begin{aligned}
{[\lambda \hat{\gamma}<\mu] } & \Longrightarrow[\rho<1] \Longrightarrow M_{r} \text { is ergodic on } \tilde{L}, \\
{[\lambda \hat{\gamma}>\mu] } & \Longrightarrow[\rho>1] \Longrightarrow M_{r} \text { is transient on } \tilde{L} .
\end{aligned}
$$

When $\lambda \hat{\gamma}<\mu$, the stationary distribution of $M_{r}$ is $\pi_{\rho, r}=(1-\rho) p_{\rho, r}$. The corresponding stationary departure process is a Poisson process of rate $\rho \mu$.

The proofs of Lemma 5.4, Theorem 5.1, and Theorem 5.3 are easily adapted to obtain Proposition 6.1. 


\subsection{Five illustrating examples}

We study five particular zero-automatic queues to illustrate the above results. We focus on three aspects: the stability region, the value of the load $\rho$, and the existence of several stationary regimes (for the queues built on zero-automatic triples instead of plain triples). When the model is simple enough, the TTE can be solved explicitly to obtain closed form formulas as for the group $\mathbb{Z} / 3 \mathbb{Z} \star \mathbb{Z} / 3 \mathbb{Z}$ discussed below. In all cases, and like any set of algebraic equations, the TTE can be solved with any prescribed precision.

In this section we also aim to convey the idea that zero-automatic queues ought to be pertinent in several modeling contexts, due to the flexibility in their definition. The five examples below should be interpreted having in mind the different 'types' of tasks detailed in the introduction (classical, positive/negative, 'one equals many', and 'dating agency').

6.2.1. The free product $\mathbb{Z} / 3 \mathbb{Z} \star \mathbb{Z} / 3 \mathbb{Z}$. Consider the plain triple $(\mathbb{Z} / 3 \mathbb{Z} \star \mathbb{Z} / 3 \mathbb{Z}, \Sigma=$ $\left.\left\{a, a^{2}, b, b^{2}\right\}, v\right)$, where $v(a)=v(b)=p$ and $v\left(a^{2}\right)=v\left(b^{2}\right)=q=\frac{1}{2}-p$, for $p \in\left(0, \frac{1}{2}\right)$. In [23, Section 4.2], the drift was computed and is given by

$$
\hat{\gamma}=-\frac{1}{4}+\frac{1}{4} \sqrt{16 p^{2}-8 p+5} \text {. }
$$

According to Theorem 5.2, in the stable case, the associated TTE have a unique admissible solution that we denote by $(\rho, r)$. Solving the TTE, we obtain

$$
\begin{gathered}
\hat{r}(a)=\hat{r}(b)=\frac{4 \lambda p^{2}-2 \lambda p+4 p \mu+\lambda}{4\left(4 \lambda p^{2}-2 \lambda p+\mu+\lambda\right)}=\frac{-4 p q \lambda+\lambda+4 p \mu}{4(-4 p q \lambda+\lambda+\mu)}, \\
\hat{r}\left(a^{2}\right)=\hat{r}\left(b^{2}\right)=\frac{1}{2}-\hat{r}(a), \\
\rho=2 \frac{4 \lambda^{2} p^{2}-2 \lambda^{2} p+\lambda \mu+\lambda^{2}}{4 \lambda^{2} p^{2}-2 \lambda^{2} p+4 \lambda \mu+\lambda^{2}+4 \mu^{2}} .
\end{gathered}
$$

In Figure 4(a), we show the stability region of the queue. The abscissa is $p$ and the ordinate is $\lambda / \mu$. In Figure 4(b), we plot the load $\rho$ as a function of $p$ and $t=\lambda / \mu$, for $p \in\left(0, \frac{1}{2}\right)$ and $\lambda / \mu \in(0, \min (1 / \hat{\gamma}, 6))$. Hence, $\rho$ is always smaller or equal to 1 ; see Theorem 5.1.

(a)

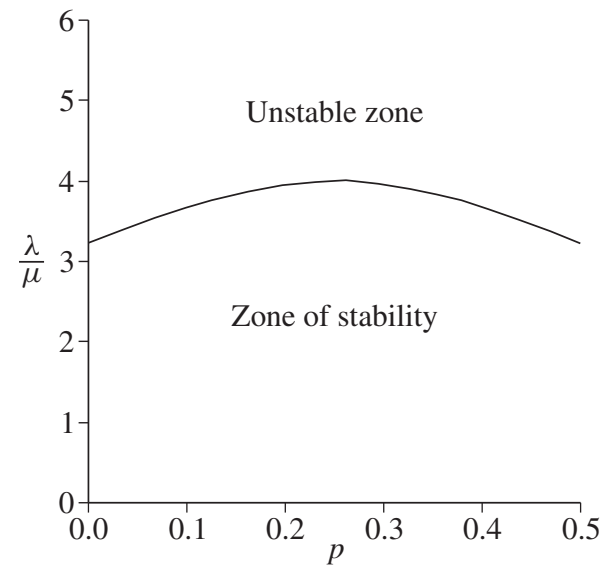

(b)

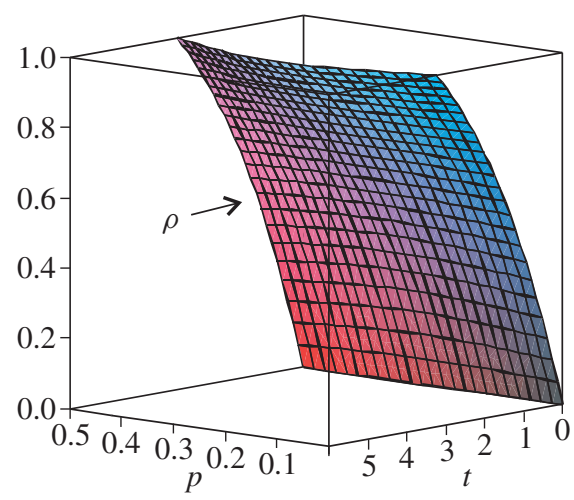

FIGURE $4: \mathbb{Z} / 3 \mathbb{Z} \star \mathbb{Z} / 3 \mathbb{Z}$ : The stability region (a) and the load $\rho$ (b). 


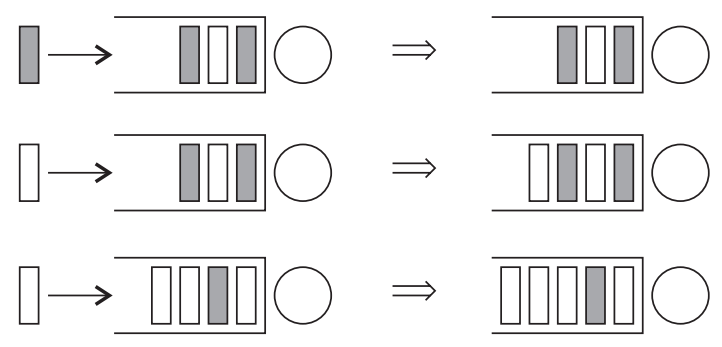

Figure 5: The queue $\mathrm{M} / \mathrm{M} /(\mathbb{N} \star \mathbb{B}, \Sigma)$ with $a$ in white and $b$ in dark gray.

(a)

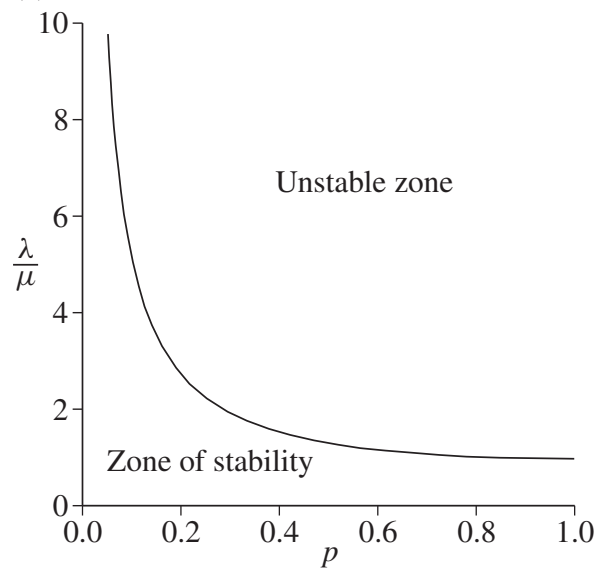

(b)

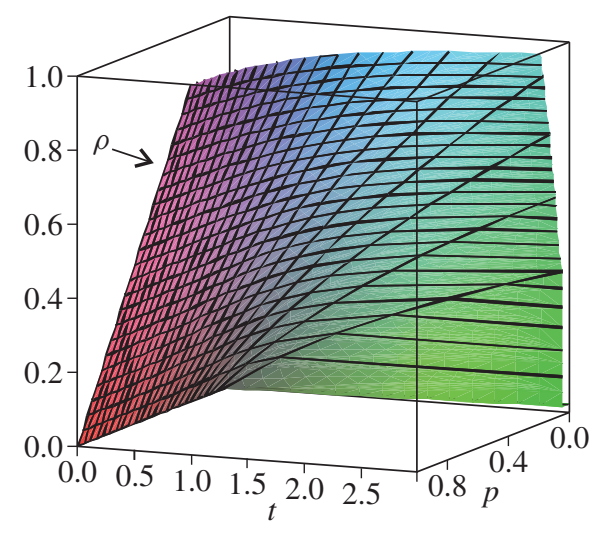

Figure 6: $\mathbb{N} \star \mathbb{B}$ : The stability region (a) and the load $\rho$ (b).

6.2.2. The free product $\mathbb{N} \star \mathbb{B}$. Consider the plain triple $\left(\{a\}^{*} \star\left\langle b \mid b^{2}=b\right\rangle, \Sigma=\{a, b\}, v\right)$, where $v(a)=p$ and $v(b)=1-p$ for $p \in(0,1)$. In Figure 5 , we illustrate the corresponding buffering mechanism. The unique solution $\hat{r}$ of the TE is $\hat{r}(c)=p, \hat{r}(b)=1-p$. The drift of the random walk is $\hat{\gamma}=(2-p) p$. According to Theorem 5.2, the associated TTE have a unique admissible solution that we denote by $(\rho, r)$. Solving the TTE, we find that $\rho$ is a solution of $f(Y)=0$, where

$$
f(Y)=\mu^{2} Y^{3}+\left(\mu^{2}+\mu \lambda+\lambda \mu p\right) Y^{2}+\left(\lambda^{2} p+\lambda \mu p\right) Y-\lambda^{2} p^{2}+\lambda^{2} p .
$$

The relation between $r(b)$ and $\rho$ is given by $\rho=[r(b)(1-p)+p] \lambda / \mu$.

In Figure 6(a), we show the stability region of the queue. The abscissa is $p$ and the ordinate is $\lambda / \mu$. In Figure 6(b), we plot the load $\rho$ as a function of $p$ and $t=\lambda / \mu$, for $p \in(0,1)$ and $\lambda / \mu \in(0, \min (1 / \hat{\gamma}, 3))$. Hence, $\rho$ is always smaller or equal to 1 ; see Theorem 5.1.

6.2.3. The free product $\mathbb{N} \star \mathbb{Z} \star \mathbb{B}$. Consider the queue associated with the plain triple $\left(\{a\}^{*} \star\right.$ $\left.F(b) \star\left\langle c \mid c^{2}=c\right\rangle, \Sigma=\left\{a, b, b^{-1}, c\right\}, v\right)$, where $v(a)=p, v(b)=v\left(b^{-1}\right)=q / 2$, and $v(c)=1-p-q$ with $p, q, p+q \in(0,1)$. The unique solution $\hat{r}$ of the associated TE is

$\hat{r}(b)=\hat{r}\left(b^{-1}\right)=\frac{1}{2}-\frac{\sqrt{1-q^{2}}}{2(1+q)}, \quad \hat{r}(a)=\frac{p(1-\hat{r}(b))}{1-\hat{r}(b)-q \hat{r}(b)}, \quad \hat{r}(c)=1-\hat{r}(a)-2 \hat{r}(b)$. 


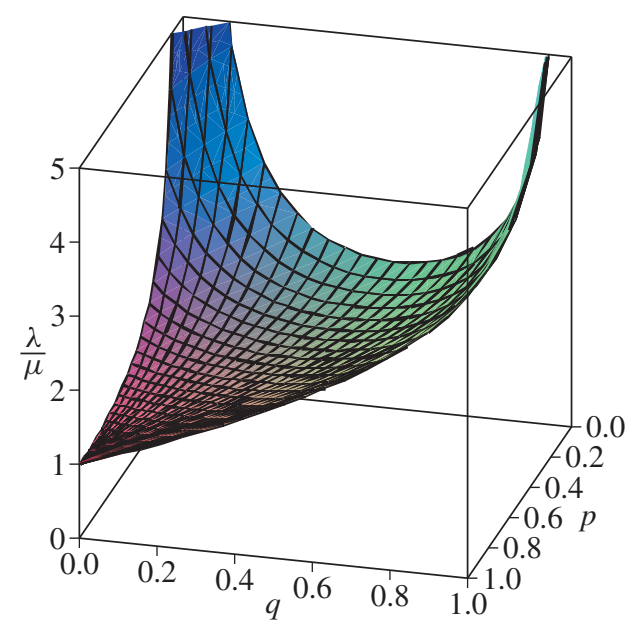

FIGURE 7: Stability region of the $\mathrm{M} / \mathrm{M} / 1 /(\mathbb{N} \star \mathbb{Z} \star \mathbb{B}, \Sigma)$ queue.

Applying Theorem 2.1, the drift of the random walk is given by $\hat{\gamma}=p+(1-p-q)(1-\hat{r}(c))$ $+q(1-2 \hat{r}(b))$. We obtain the plot in Figure 7 in which the stability region is the region below the surface.

6.2.4. The free group $\mathbb{Z}$ and the free product $\mathbb{N} \star \mathbb{Z}$. Consider the zero-automatic queue $\left(F(a),\left\{a, a^{-1}\right\}, v, r, \lambda, \mu\right)$, where $v$ is a nondegenerate probability measure on $\Sigma=\left\{a, a^{-1}\right\}$. In Figure 8, we illustrate the corresponding buffering mechanism. Such a mechanism is similar to the one of Gelenbe's G-queue. The underlying triple $\left(F(a),\left\{a, a^{-1}\right\}, v\right)$ is not plain (Definition 2.1), but it is zero-automatic (Definition 6.3). Here the graph of successors $(\Sigma, \rightarrow)$, see (2.4), is not connected. Also, the random walk $(X, v)$ is not transient but is null-recurrent when $v(a)=v\left(a^{-1}\right)=\frac{1}{2}$. The drift of the random walk is easily computed and given by

$$
\hat{\gamma}=\left|v(a)-v\left(a^{-1}\right)\right|
$$

Assume first that $v(a)=v\left(a^{-1}\right)$. Solving the TTE, we find that $(\lambda /(2 \mu+\lambda), r)$ is a solution for all $r \in \overline{\mathscr{B}}$. This means that the queue is stable and has a product form distribution under any boundary condition. This interesting behavior can be traced back to the fact that the random walk $(X, v)$ is not transient.

Now assume that $v(a) \neq v\left(a^{-1}\right)$. There are two possible solutions for the TTE, given by

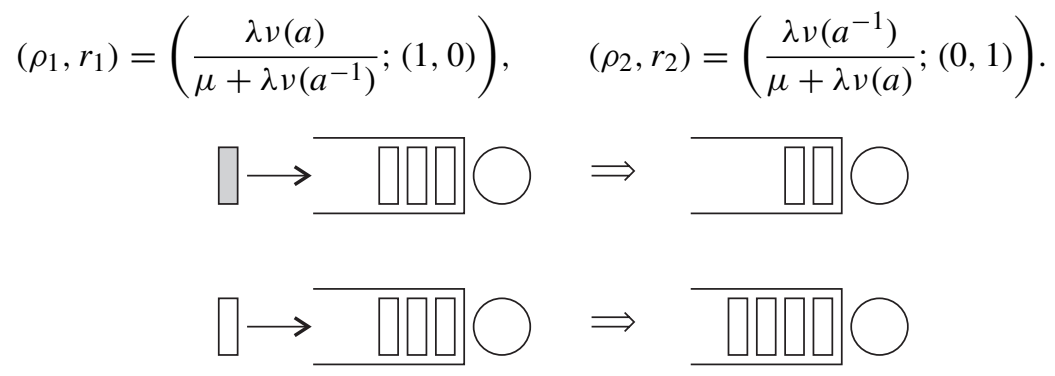

FIGURE 8: The M/M/1/( $F(a), \Sigma)$ queue with $a$ in white and $a^{-1}$ in light gray. 


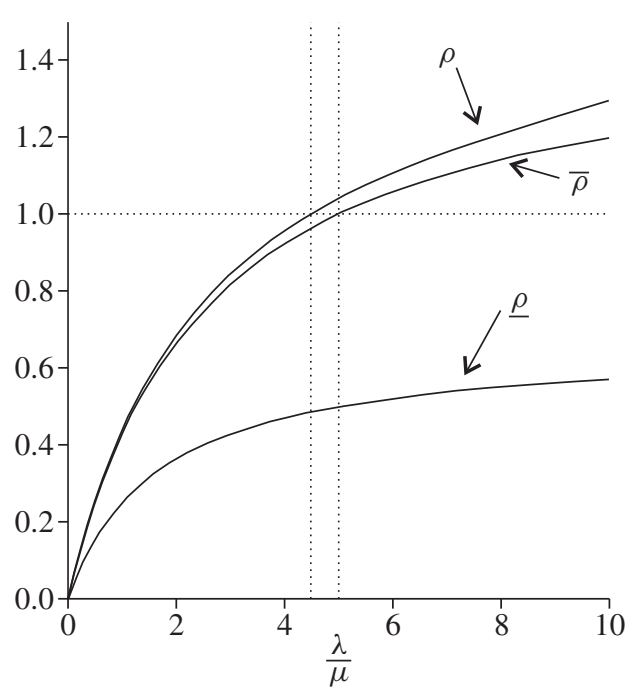

Figure 9: $F(a)$ and $F(a) \star\{c\}^{*}:$ the loads $\rho, \bar{\rho}$, and $\underline{\rho}$ plotted as a function of $\lambda / \mu$.

The two solutions correspond to extremal values for $r$, which means that in the buffer there is only one type of customer with probability 1 , i.e. if $r_{1}=(1,0)$ then there is only $a$ in the buffer, if $r_{2}=(0,1)$ then there is only $a^{-1}$ in the buffer. Here we recover a model very close to the classical G-queue. Let $\underline{\rho}=\min \left\{\rho_{1}, \rho_{2}\right\}$ and $\bar{\rho}=\max \left\{\rho_{1}, \rho_{2}\right\}$, and define $\underline{r}$ and $\bar{r}$ accordingly. We have

$$
\underline{\rho}<1, \quad[\bar{\rho}<1] \Longleftrightarrow[\lambda \hat{\gamma}<\mu]
$$

The stationary distribution of the zero-automatic queue $(F(a), \Sigma, v, \underline{r}, \lambda, \mu)$ is

$$
\pi_{\underline{r}}\left(1_{\Sigma^{*}}\right)=1-\underline{\rho}, \quad \pi_{\underline{r}}\left(x^{n}\right)=(1-\underline{\rho}) \underline{\rho}^{n}, \quad \text { for all } n \geq 1,
$$

where $x=a$ if $v(a)<v\left(a^{-1}\right)$, and $x=a^{-1}$ if $v(a)>v\left(a^{-1}\right)$. When $\lambda \hat{\gamma}<\mu$, the zeroautomatic queue $(F(a), \Sigma, \nu, \bar{r}, \lambda, \mu)$ also has a product form stationary distribution of the form (6.5) with $\bar{\rho}$ instead of $\underline{\rho}$.

Consider now a boundary condition $r \in \mathscr{B}$. In particular, $r \neq r_{1}, r \neq r_{2}$, so that the stationary distribution is not of product form. However, if $\lambda \hat{\gamma}<\mu$, then the stationary distribution $\pi_{r}$ can still be determined explicitly by solving the global balance equations. It is given by

$$
\begin{gathered}
\pi_{r}\left(1_{\Sigma^{*}}\right)=\left(1+r(a) \frac{\rho_{1}}{1-\rho_{1}}+r\left(a^{-1}\right) \frac{\rho_{2}}{1-\rho_{2}}\right)^{-1}, \\
\pi_{r}\left(a^{n}\right)=\pi_{r}\left(1_{\Sigma^{*}}\right) r(a) \rho_{1}^{n}, \quad \pi_{r}\left(a^{-n}\right)=\pi_{r}\left(1_{\Sigma^{*}}\right) r\left(a^{-1}\right) \rho_{2}^{n},
\end{gathered}
$$

where $\rho_{1}$ and $\rho_{2}$ are defined in (6.4). The expression in (6.6) is 'almost' of product form. So, why do we prefer an expression like the one in (6.5)? The point is that the departure process associated with a stationary distribution of type (6.6) is not Poisson, as opposed to the one associated with (6.5). Having a Poisson departure process is crucial to build product form networks; see [9].

To summarize, when $\lambda \hat{\gamma}<\mu$, there are two variants of the zero-automatic queue with a product form. We would like to argue that one of the two makes more 'physical' sense. To 
this end, consider the plain triple $\left(F(a) \star\{c\}^{*},\left\{a, a^{-1}, c\right\}, v\right)$ with $0<v(c) \ll 1$. According to Theorem 5.2, there exists a single variant of the queue with a product form. Let $\rho$ be the corresponding load. We want to determine which one of the two solutions in (6.4) is recovered when letting $v(c)$ tend to zero. As the TTE are difficult to solve explicitly, we content ourselves with numerical evidence. In Figure 9, we plot $\rho, \bar{\rho}$, and $\rho$ as functions of $\lambda / \mu$, for $\nu(c)=0.01$ and $\nu(a)=p=\frac{3}{5}$. We see that $\rho$ tends to the larger solution $\bar{\rho}$. The two vertical lines delimit two stability regions (with and without $\{c\}$ ). They have an abscissa equal to the inverse of the drift $\hat{\gamma}^{-1}$ for the random walk on $F(a) \star\{c\}^{*}$ and $F(a)$ respectively.

6.2.5. The monoid $M=\langle a, b \mid a b=1\rangle$. Consider the bicyclic monoid $M=\langle a, b \mid a b=1\rangle$. Here we have a new 'type' of task. It is close to the positive/negative type but with no symmetry between the positive and negative customers. Consider the triple $(M, \Sigma=\{a, b\}, v)$, with $v(a)=p \in(0,1), v(b)=1-p$. This is a zero-automatic triple but not a plain triple. In particular, the graph of successors $(\Sigma, \rightarrow)$ is not strongly connected. The drift of the random walk is easily computed and given by $\hat{\gamma}=|1-2 p|$. Solving the associated TTE, we find that there is one solution if $p \leq \frac{1}{2}$ and two solutions if $p>\frac{1}{2}$. More precisely, these two solutions are

$$
\begin{aligned}
& \left(\rho_{1}, r_{1}\right)=\left(\frac{\lambda(1-p)}{\mu+\lambda p} ;(0,1)\right) \text { for all } p \\
& \left(\rho_{2}, r_{2}\right)=\left(\frac{\lambda p}{\mu+\lambda(1-p)} ;\left(\frac{2 p-1}{p}, \frac{1-p}{p}\right)\right) \quad \text { if } p>\frac{1}{2}
\end{aligned}
$$

We have

$$
\begin{gathered}
\text { if } p \leq \frac{1}{2}, \quad\left[\rho_{1}<1\right] \Longleftrightarrow[\lambda \hat{\gamma}<\mu], \\
\text { if } p>\frac{1}{2}, \quad \text { and if } \rho_{1}<1, \quad\left[\rho_{2}<1\right] \Longleftrightarrow[\lambda \hat{\gamma}<\mu] .
\end{gathered}
$$

In Figure 10, we show the solutions to the TTE as a function of $p$ and $\lambda / \mu$.

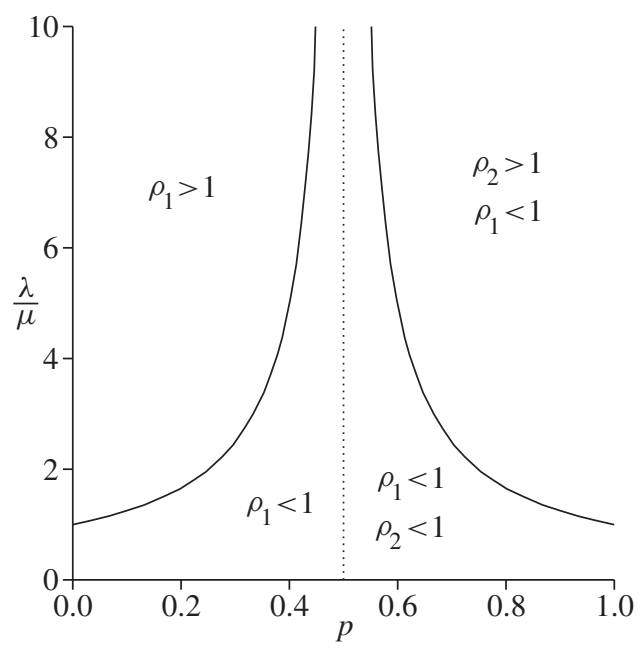

FIgURE 10: $F(a)$ : the solutions to the TTE. 

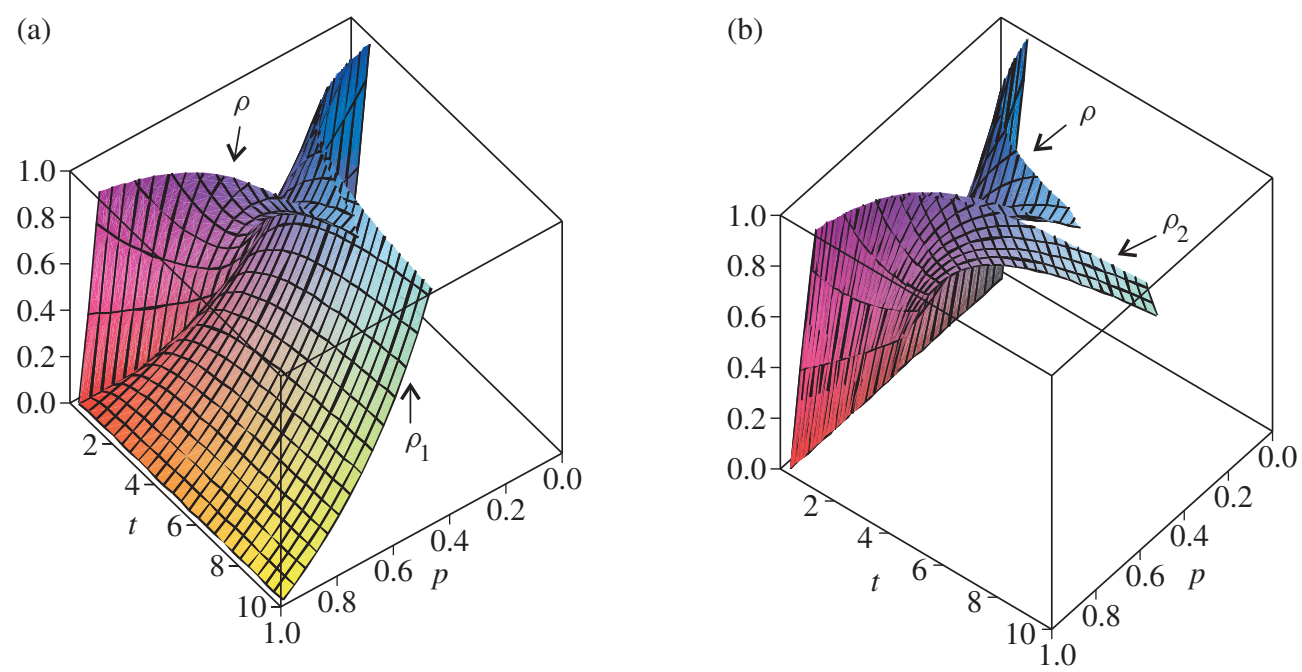

FiguRe 11: $\langle a, b \mid a b=1\rangle$ and $\langle a, b \mid a b=1\rangle \star\{c\}^{*}:$ the loads $\rho$ and $\rho_{1}$ (part (a)) and $\rho$ and $\rho_{2}$ (part (b)) plotted as functions of $v(a)$ and $t=\lambda / \mu$.

To discriminate between $\left(\rho_{1}, r_{1}\right)$ and $\left(\rho_{2}, r_{2}\right)$, we proceed as for $F(a)$. Consider $X=$ $\langle a, b \mid a b=1\rangle \star\{c\}^{*}$. Let $v(a)=p, v(b)=q$, and $v(c)=1-p-q$ with $p, q>0$, $p+q<1$. The triple $(X,\{a, b, c\}, v)$ is zero-automatic. The TE can be solved explicitly. It turns out that there is a unique solution $\hat{r}$ which is determined by

$$
\hat{r}(a)=\frac{p(1-\hat{r}(b))}{1-p \hat{r}(b)}, \quad \hat{r}(b)=\frac{1-\sqrt{1-4 p q}}{2 p}, \quad \hat{r}(c)=\frac{1-p-q}{1-p \hat{r}(b)} .
$$

According to Theorem 2.1, the corresponding drift is $\hat{\gamma}=\sqrt{1-4 p q}$. Now, solving the TTE, we find a unique admissible solution $(\rho, r)$ given by

$$
\rho=\frac{\lambda(1-\operatorname{pr}(b))}{\mu+\lambda \operatorname{pr}(b)}, \quad r=\hat{r} .
$$

We have $\rho<1$ if and only if $\mu>\lambda \hat{\gamma}=\lambda \sqrt{1-4 p q}$. Let us observe what happens when $v(c)$ tends to zero. When $p<\frac{1}{2}$, there is only one solution $\left(\rho_{1}, r_{1}\right)$ to the TTE of the first case (without $\{c\})$. And, as expected, $(\rho, r)$ tends to $\left(\rho_{1}, r_{1}\right)$ when $v(c) \rightarrow 0$. When $p>\frac{1}{2}$, there are two possible solutions $\left(\rho_{1}, r_{1}\right)$ and $\left(\rho_{2}, r_{2}\right)$ to the TTE of the first case. In this case, $(\rho, r)$ tends to $\left(\rho_{2}, r_{2}\right)$ when $v(c) \rightarrow 0$. In Figure 11(a), we plot $\rho$ and $\rho_{1}$ as functions of $p$ and $\lambda / \mu$, for $v(c)=0.01$. In Figure 11(b), we plot $\rho$ and $\rho_{2}$.

\section{References}

[1] Aliprantis, C. And Border, K. (1999). Infinite Dimensional Analysis: A Hitchhiker's Guide, 2nd edn. Springer, Berlin.

[2] Asmussen, S. (1987). Applied Probability and Queues. John Wiley, Chichester.

[3] Baccelli, F. And Foss, S. (1995). On the saturation rule for the stability of queues. J. Appl. Prob. 32, 494-507.

[4] Brémaud, P. (1999). Markov Chains: Gibbs Fields, Monte Carlo Simulation, and Queues (Texts Appl. Math. 31). Springer, New York.

[5] Chao, X. and Miyazawa, M. (2000). Queueing networks with instantaneous movements: a unified approach by quasi-reversibility. Adv. Appl. Prob. 32, 284-313. 
[6] Chao, X., Miyazawa, M. and Pinedo, M. (1999). Queueing Networks. Customers, Signals, and Product Form Solutions. John Wiley, New York.

[7] Cohen, J. W. (1982). The Single Server Queue, 2nd edn. North-Holland, Amsterdam.

[8] Dao-Thi, T.-H. AND MaIResse, J. (2005). Zero-automatic queues. In Formal Techniques for Computer Systems and Business Processes LNCS 3670, Springer, Berlin, pp. 64-78.

[9] Dao-Thi, T.-H. And Mairesse, J. (2006). Zero-automatic networks. In Proc. VALUETOOLS (Pisa, Italy), ACM, New York.

[10] Dayar, T. And Quessette, F. (2002). Quasi-birth-and-death processes with level-geometric distribution. SIAM J. Matrix Anal. Appl. 24, 281-291.

[11] Dynkin, E. And Malyutov, M. (1961). Random walk on groups with a finite number of generators. Soviet Math. Dokl. 2, 399-402.

[12] Epstein, D. et al. (1992). Word Processing in Groups. Jones and Bartlett, Boston, MA.

[13] Fourneau, J.-M., Gelenbe, E. And Suros, R. (1996). G-networks with multiple classes of negative and positive customers. Theoret. Comput. Sci. 155, 141-156.

[14] Gelenbe, E. (1991). Product-form queueing networks with negative and positive customers. J. Appl. Prob. 28, 656-663.

[15] Gelenbe, E. ANd Pujolle, G. (1998). Introduction to Queueing Networks, 2nd edn. John Wiley, Chichester.

[16] Guivarc'H, Y. (1980). Sur la loi des grands nombres et le rayon spectral d'une marche aléatoire. Astérisque 74, 47-98.

[17] Kelly, F. (1979). Reversibility and Stochastic Networks. John Wiley, New York.

[18] Kingman, J. (1973). Subadditive ergodic theory. Ann. Prob. 1, 883-909.

[19] He, Q.-M. (2003). The classification of matrix GI/M/1-type Markov chains with a tree structure and its applications to queueing. J. Appl. Prob. 40, 1087-1102.

[20] Latouche, G. And Ramaswami, V. (1999). Introduction to Matrix Analytic Methods in Stochastic Modeling. SIAM, Philadelphia, PA.

[21] Ledrappier, F. (2001). Some asymptotic properties of random walks on free groups. In Topics in Probability and Lie Groups: Boundary Theory (CRM Proc. Lecture Notes 28), American Mathematical Society, Providence, RI, pp. 117-152.

[22] Mairesse, J. (2005). Random walks on groups and monoids with a Markovian harmonic measure. Electron. J. Prob. 10, 1417-1441.

[23] Mairesse, J. And Mathéus, F. (2007). Random walks on free products of cyclic groups. J. London Math. Soc. 75, 47-66.

[24] Neuts, M. (1981). Matrix-Geometric Solutions in Stochastic Models: An Algorithmic Approach. Johns Hopkins University Press, Baltimore, MD.

[25] Sawyer, S. and Steger, T. (1987). The rate of escape for anisotropic random walks in a tree. Prob. Theory Relat. Fields 76, 207-230.

[26] Serfozo, R. (1999). Introduction to Stochastic Networks. Springer, Berlin.

[27] Stallings, J. (1966). A remark about the description of free products of groups. Proc. Camb. Philos. Soc. 62, 129-134.

[28] Yeung, R. And Sengupta, B. (1994). Matrix product-form solutions for Markov chains with a tree structure. Adv. Appl. Prob. 26, 965-987. 